Towards a Scalable Fully-Implicit Fully-coupled Resistive MHD Formulation with Stabilized FE Methods

J. N. Shadid, R. P. Pawlowski, J. W. Banks, L. Chacon, P. T. Lin, R. S. Tuminaro

June 11, 2009

Journal of Computational Physics 
This document was prepared as an account of work sponsored by an agency of the United States government. Neither the United States government nor Lawrence Livermore National Security, LLC, nor any of their employees makes any warranty, expressed or implied, or assumes any legal liability or responsibility for the accuracy, completeness, or usefulness of any information, apparatus, product, or process disclosed, or represents that its use would not infringe privately owned rights. Reference herein to any specific commercial product, process, or service by trade name, trademark, manufacturer, or otherwise does not necessarily constitute or imply its endorsement, recommendation, or favoring by the United States government or Lawrence Livermore National Security, LLC. The views and opinions of authors expressed herein do not necessarily state or reflect those of the United States government or Lawrence Livermore National Security, LLC, and shall not be used for advertising or product endorsement purposes. 


\title{
Towards a Scalable Fully-Implicit Fully-coupled Resistive MHD Formulation with Stabilized FE Methods *
}

\author{
J.N. Shadid ${ }^{1}$, R.P. Pawlowski ${ }^{1}$, J.W. Banks ${ }^{2}$, L. Chacón ${ }^{3}$, P.T Lin ${ }^{1}$, \\ R.S. Tuminaro ${ }^{1}$
}

\begin{abstract}
This paper presents an initial study that is intended to explore the development of a scalable fully-implicit stabilized unstructured finite element (FE) capability for low-Mach-number resistive MHD. The discussion considers the development of the stabilized FE formulation and the underlying fully-coupled preconditioned Newton-Krylov nonlinear iterative solver. To enable robust, scalable and efficient solution of the large-scale sparse linear systems generated by the Newton linearization, fully-coupled algebraic multilevel preconditioners are employed. Verification results demonstrate the expected order-of-acuracy for the stabilized FE discretization of a $2 \mathrm{D}$ vector potential form for the steady and transient solution of the resistive MHD system. In addition, this study puts forth a set of challenging prototype problems that include the solution of an MHD Faraday conduction pump, a hydromagnetic Rayleigh-Bernard linear stability calculation, and a magnetic island coalescence problem. Initial results that explore the scaling of the solution methods are presented on up to 4096 processors for problems with up to 64M unknowns on a CrayXT3/4. Additionally, a large-scale proof-of-capability calculation for 1 billion unknowns for the MHD Faraday pump problem on 24,000 cores is presented.
\end{abstract}

\section{Introduction}

The magnetohydrodynamics (MHD) model describes the dynamics of charged fluids in the presence of electromagnetic fields. MHD models are used to describe important phenomena in the natural world (e.g., solar flares, astrophysical magnetic field generation, Earth's magnetosphere interaction with the solar wind) and in technological applications (e.g., spacecraft propulsion, magnetically confined plasma for fusion energy devices such as tokamak reactors and plasma dynamics in pulsed reactors such as Zpinch devices) [1]. The mathematical basis for the continuum modeling of these systems is the solution of the governing partial differential equations (PDEs) describing conservation of mass, momentum,

\footnotetext{
* This work was partially supported by DOE NNSA ASC Algorithms effort, the DOE Office of Science AMR program at Sandia National Laboratory under contract DE-AC04-94AL85000 and Lawrence Livermore National Laboratory under contract DE-AC52-07NA27344.

1 Sandia National Laboratories, Albuquerque, New Mexico, 87185

2 Lawrence Livermore National Laboratory, Livermore, California , 94551

3 Oak Ridge National Laboratory, Oak Ridge, TN 37831
} 
charge, and thermal energy augmented by Maxwell's equations for the electric and magnetic field. This system of PDEs is non-self adjoint, strongly coupled, highly nonlinear and characterized by multiple physical phenomena that span a very large range of length- and time-scales. These interacting, nonlinear multiple time-scale physical mechanisms can balance to produce steady-state behavior, nearly balance to evolve a solution on a dynamical time scale that is long relative to the component time-scales, or can be dominated by just a few fast modes. These characteristics make the scalable, robust, accurate, and efficient computational solution of these systems over relevant dynamical time scales of interest (or to steady-state solutions) extremely challenging.

For multiple-time-scale systems, fully-implicit methods can be an attractive choice that can often provide unconditionally-stable time integration techniques [2,3]. The stability of these methods, however, comes at a very significant price, as these techniques generate large and highly nonlinear sparse systems of equations that must be solved at each time step. For this reason, the dominant computational solution strategy has been the use of explicit [4-9] and partially implicit methods that include implicit-explicit [10-14], semi-implicit [15-19], and operator-splitting [20,21] time integration methods. With the exception of fully-explicit strategies that are limited by stability restrictions to follow the fastest component time scale, these temporal integration methods all include some implicit aspects of time integration to allow more efficient solution of multiple-time-scale MHD systems. The implicitness of these algorithms is intended to remove a source of numerical stiffness in the problem, either parabolic diffusion stability constraints or fast hyperbolic wave phenomena such as those introduced by Hall physics $[22,23]$. While these types of techniques currently form the basis for most production level resistive MHD simulation tools (see e.g. $[19,13]$ ) there are a number of outstanding numerical and computational issues. These include conditional stability limits, operator-splitting-type errors, and limited temporal order of accuracy $[17,18]$.

Recently progress has been made in developing fully-implicit formulations that attempt to robustly and accurately integrate these systems and follow the dynamical time-scales of interest $[24,25,11,10,26,27,22,23,28$, In Ref. [24], a nonlinear implicit MHD solver is proposed based on a implicit-operator-split (IOS) approach. The IOS algorithm employs Krylov solvers for required inversions in each split step, and is iterated upon in a Gauss-Seidel manner to achieve some degree of nonlinear consistency. However, for large implicit time steps, large numerical errors are reported possible in transient calculations with this algorithm unless enough nonlinear iterations are taken [24]. In Refs. [11,10], incomplete-LUpreconditioned Krylov methods are employed to invert the linearly implicit (and also implicit-explicit) set of MHD equations in the context of the Versatile Advection Code. CPU speedups vs. explicit of $\sim 40$ are reported [10]. An unpreconditioned Newton-Krylov solver for 3D compressible MHD is explored in Ref. [26], which also reports order-of-magnitude speedups vs. explicit approaches for fine enough grids. More recently, the same researchers have developed an "operator-based" parallel preconditioner for 3D MHD, based on directional splitting of the implicit operator and followed by a characteristic decomposition of the resulting directional PDE operators [30]. Reference [27] explores a Newton-Krylov-Schwarz parallel approach for the reduced Hall MHD model, where gains of an order of magnitude with respect to explicit approaches and good parallel scalability are reported. Finally, Refs. $[22,23,28,29]$ develop optimal "physics-based" preconditioning strategies for a fully implicit Newton-Krylov treatment of 2D and 3D extended MHD, and report excellent parallel scalability and algorithmic speedups ranging from one to two orders of magnitude.

The study presented in this paper is intended to complement the work above by considering the development of methods that are capable of enabling robust and efficient direct-to-steady-state and fully-implicit fully-coupled solution methods for resistive MHD based on a stabilized FE formulation. This formulation relies on an inexact Newton-Krylov solution method [31,32] to solve the large-scale nonlinear algebraic systems. The preconditioning methods are based on a variable-overlap additive one- 
level Schwarz preconditioner [33,34] and a relatively new algebraic multilevel technique that employs a graph-based aggressive-coarsening aggregation method applied to the nonzero block structure of the Jacobian matrix $[35,36]$. The algebraic multilevel method effectively uses corrections that are computed by a sequence of coarse operators to accelerate the convergence of the iterative Krylov method on the fine mesh.

In the context of stabilized finite element methods, Salah et. al. [37] developed a formulation for the constant resistivity magnetic induction equation with a given velocity field. They use a Lagrange multiplier to enforce the solenoidal constraint on the magnetic field, $\mathbf{B}$ and thus develop a system with four unknowns, the three components of $\mathbf{B}$ and the Lagrange multiplier, $r$. This system requires compatible spaces for $\mathbf{B}$ and $r$ that respect the Ladyzhenskaya-Babuska-Brezzi (LLB) condition, that is analogous to the velocity and pressure spaces $(\mathbf{v}, p)$ in the Stokes flow system (see e.g. [38,39]). An inconsistent stabilized formulation [40] following Brezzi and Pitkaranta [41] is used to allow equal order interpolation for $\mathbf{B}$ and $r$. These methods were subsequently extended to an incompressible, constant resistivity MHD system with both a Lagrange multiplier formulation for $\mathbf{B}$ as described above, and a vector potential formulation [42]. The solution of the resistive MHD system uses an outer decoupled nonlinear solution strategy. This decoupled strategy solves the flow and magnetics system separately in each sub-step and couples the system by the outer iteration that can sub-cycle the component solves. An ILU preconditioned Newton-Krylov type solver is used for the flow equations and a direct sparse solver is used for the linear magnetics equation. Codina and Silva [43] developed a stabilized FE formulation for resistive MHD, in the curl form of the equations with constant properties, and develop stability parameters that handle the velocity-pressure coupling, the solenoidal constraint by a Lagrange multiplier method, and streamline upwind Petrov-Galerkin (SUPG) like terms that control oscillation due to convection effects. A fixed point nonlinear solution is employed to resolve the nonlinearities and no mention is made of the component linear solver(s) that are used. These authors present a coercivity result for the system that enables development of the stabilization parameters. Other studies of stabilized FE methods applied to resistive MHD systems include Gerbeau [44] that considered the coercivity of a stabilized FE formulation for steady state systems and employed a fixed-point (Picard) type nonlinear solution strategy, Lankalapalli et. al. [45] developed a vorticity-streamfunction vector potential formulation that uses a SUPG FE discretization and a fixed-point nonlinear solver with a GMRES iterative method.

Our discretization of the governing resistive MHD equations employs stabilized finite element (FE) methods based on the general developments of Hughes et. al.(see e.g. [46-52]). The stabilized formulation circumvents the LBB condition for compatible discretization for mixed finite element formulations of the saddle point problem arising from discretization of the incompressible MHD equations. The stabilized FE formulation allows for equal order interpolation of the incompressible MHD equations without spurious pressure modes and reduces oscillations in the Galerkin FE formulation for highly-convected flows. In addition, to enforce the divergence-free condition of the magnetic field, a vector-potential formulation of the resistive MHD system is used. This formulation implements a simplified form of a consistently stabilized FE method [40]. In the current context of low Mach number resistive MHD systems, the benefit in addition to solvability of the system, is that the use of equal order interpolation simplifies the data structures of a parallel unstructured FE code and the linear algebra interface for the iterative solution methods that are employed, as described below.

The remainder of this paper is organized as follows. Section 2 presents a brief summary of the resistive MHD equations and a vector potential form for magnetics effects. The stabilized FE formulation of the governing 2D vector potential form of the resistive MHD equations is presented in Section 3. In Section 4 a brief overview of the fully-implicit Newton-Krylov solution method is presented with a discussion of the domain decomposition and multilevel preconditioners. In Section 5 we present representative 
verification, order-of-accuracy results and representative performance, scaling and simulation results of these solution methods for some illustrative resistive MHD problems. Finally in Section 6 we close with a few conclusions.

\section{MHD Equations and the Simplified 2D Vector Potential Form}

A base model for MHD that includes transport effects is the one-fluid visco-resistive MHD system [53,1]. From a continuum perspective, this system of governing equations includes the Navier-Stokes equations augmented by the Lorentz force term in the momentum equation and a Joule heating term included in an internal energy equation as in (1) - (3).

$$
\begin{gathered}
\frac{\partial(\rho \mathbf{v})}{\partial t}+\nabla \cdot[\rho \mathbf{v} \otimes \mathbf{v}-\mathbf{T}]-\mathbf{J} \times \mathbf{B}=\mathbf{0} \\
\frac{\partial \rho}{\partial t}+\nabla \cdot[\rho \mathbf{v}]=0 \\
\frac{\partial(\rho e)}{\partial t}+\nabla \cdot[\rho \mathbf{v} e+\mathbf{q}]-\mathbf{T}: \nabla \mathbf{v}-\eta\|\mathbf{J}\|^{2}+Q=0 .
\end{gathered}
$$

In this base model the fluid system equations are closed with the assumed form of the constitutive equations for the Newtonian fluid stress tensor, T, and the Fourier heat flux vector, q,

$$
\begin{gathered}
\mathbf{T}=-P \mathbf{I}+\mathbf{\Pi}=-\left(P+\frac{2}{3} \mu(\nabla \cdot \mathbf{v})\right) \mathbf{I}+\mu\left[\nabla \mathbf{v}+\nabla \mathbf{v}^{T}\right], \\
\mathbf{q}=-\lambda \nabla T,
\end{gathered}
$$

and the assumption of a thermodynamically simple material equation of state:

$$
\begin{aligned}
& P=\hat{P}(e, \rho) \\
& T=\hat{T}(e, \rho) .
\end{aligned}
$$

In the case of resistive MHD, a simplified form of Maxwell's equations that includes Faraday's law, Ampere's law neglecting the displacement current, and a simple resistive Ohm's law for the electric field [1],

$$
\begin{array}{r}
\frac{\partial \mathbf{B}}{\partial t}-\nabla \times \mathbf{E}=\mathbf{0} \\
\mathbf{J}-\frac{1}{\mu_{0}} \nabla \times \mathbf{B}=\mathbf{0} \\
\mathbf{E}+\mathbf{v} \times \mathbf{B}-\eta \mathbf{J}=\mathbf{0}
\end{array}
$$

is used to obtain the magnetic field evolution equation of the form:

$$
\frac{\partial \mathbf{B}}{\partial t}-\nabla \times(\mathbf{v} \times \mathbf{B})+\nabla \times\left(\frac{\eta}{\mu_{0}} \nabla \times \mathbf{B}\right)=\mathbf{0}
$$

In the next subsections, the system (1) - (6) is specialized to a formulation that allows a low-order FE interpolation of the velocity field $\mathbf{v}$, the pressure $P$, the temperature, $T$, and the magnetic flux, $\mathbf{B}$, derived from a magnetic vector potential, $\mathbf{A}$. 


\subsection{A Vector Potential Formulation}

In this section an alternate formulation based on the classical scalar and vector potential formulation [54] is introduced that simplifies the form of the resulting evolution equations to an unsteady convectiondiffusion-reaction type equation. Further, this form in $2 \mathrm{D}$ permits the $\left(B_{x}, B_{y}\right)$ components in the plane of the magnetic flux to be computed with one component of the vector potential $A_{z}$. In the continuum, Maxwell's equations enforce the involution $\nabla \cdot \mathbf{B}=0$, and thus a vector potential, $\mathbf{A}$, exists such that $\mathbf{B}=\nabla \times \mathbf{A}$. The electric field can be defined in terms of the vector potential and a scalar potential, $\phi$ [54]:

$$
\mathbf{E}=-\nabla \phi-\frac{\partial \mathbf{A}}{\partial t}
$$

Using Ohm's law for the electric field and assuming a Coulomb-type gauge [54] defined by

$$
\phi+\frac{\eta}{\mu_{0}} \nabla \cdot \mathbf{A}=0
$$

the vector potential evolution equation can be shown to reduce to:

$$
\frac{\partial \mathbf{A}}{\partial t}-\mathbf{v} \times(\nabla \times \mathbf{A})-\frac{\eta}{\mu_{0}} \nabla^{2} \mathbf{A}-\frac{1}{\mu_{0}}[\nabla \eta](\nabla \cdot \mathbf{A})+\mathbf{E}^{0}=0,
$$

where an externally applied electric field, $\mathbf{E}^{0}$, has been included. This equation defines the vector potential, A, and therefore the magnetic flux, B, up to the arbitrary scalar potential $\phi$ that can be recovered if desired from Eqn (8). In two dimensions the vector potential reduces to $\mathbf{A}=\left(0,0, A_{z}\right)$ and evolution equation (9) becomes

$$
\frac{\partial A_{z}}{\partial t}+\mathbf{v} \cdot \nabla A_{z}-\frac{\eta}{\mu_{0}} \nabla^{2} A_{z}+E_{z}^{0}=0
$$

for a single scalar field $A_{z}$. This equation exhibits a standard convection diffusion form that is convenient for developing stabilized FE formulations. Additionally, this formulation includes all the physical mechanisms and therefore all the representative time-scales of the complete resistive MHD system. As such, the use of the $2 \mathrm{D}$ vector potential form of the equations provides a very convenient and efficient initial formulation for studying the development of scalable fully-implicit fully-coupled stabilized FE formulations for resistive MHD.

It should be noted that development of Eqn. (10) required $\nabla \cdot \mathbf{B}=\nabla \cdot \nabla \times \mathbf{A}=0$ that is satisfied identically by the continuum solution of Maxwell's equations. This condition is not necessarily satisfied in all discrete approximations. In the case of linear nodal elements in 2D, this property can be shown to hold on element interiors. However the non-continuity of the normal derivatives at element edges in a $C^{0} \mathrm{FE}$ approximation allows violation of this condition on the skeleton of the $\mathrm{FE}$ mesh, which is a set of measure zero. This implies that the divergence condition holds point-wise on element interiors and in an $L_{2}$ sense over any finite sub-region of the domain. In addition it can be shown that at element edges $\mathbf{B} \cdot \hat{\mathbf{n}}$ is continuous, a condition that is also required on any surface in the continuous problem. In $3 \mathrm{D}$, linear nodal elements do not satisfy the divergence free condition on element interiors and the benefit of this approach is therefore not as clear. The necessity of satisfying the solenoidal involution, to machine precision, for various regimes of MHD modeling is a topic of considerable current interest in both structured and unstructured mesh finite volume and unstructured finite element contexts $[9,55,56]$

Finally, the desire to use low order FE basis functions for the vector potential necessitates the consideration of the representation of the Lorentz force term in the momentum equation and the Joule 


\begin{tabular}{|l|c|}
\hline Momentum & $\mathbf{R}_{m}=\rho \frac{\partial \mathbf{v}}{\partial t}+\rho(\mathbf{v} \cdot \nabla \mathbf{v})+\nabla \cdot\left(-\frac{1}{\mu_{0}} \mathbf{B} \otimes \mathbf{B}-\mathbf{\Pi}+\left(P+\frac{1}{2 \mu_{0}}\|\mathbf{B}\|^{2}\right) \mathbf{I}\right)$ \\
\hline Total Mass & $R_{P}=\frac{\partial \rho}{\partial t}+\nabla \cdot(\rho \mathbf{v})$ \\
\hline $\begin{array}{l}\text { Thermal En- } \\
\text { ergy }\end{array}$ & $R_{T}=\rho \hat{C}_{p} \frac{\partial T}{\partial t}+\rho \hat{C}_{p}(\mathbf{v} \cdot \nabla T)+\nabla \cdot \mathbf{q}-\eta\|\mathbf{J}\|^{2}$ \\
\hline $\begin{array}{l}\text { 2D Vector } \\
\text { Potential Eq. }\end{array}$ & $R_{A_{z}}=\frac{\partial A_{z}}{\partial t}+\mathbf{v} \cdot \nabla A_{z}-\frac{\eta}{\mu_{0}} \nabla^{2} A_{z}+E_{z}^{0}$ \\
& $\mathbf{B}=\nabla \times \mathbf{A} ; \quad \mathbf{A}=\left(0,0, A_{z}\right)$ \\
\hline
\end{tabular}

Table 1

Residual form of governing low Mach number resistive MHD equations with the 2D form of the vector potential evolution equation in advection-diffusion form. The primitive variables are the velocity vector $\mathbf{u}$, the hydrodynamic pressure $P$, the temperature $T$, and the $A_{z}$ component of the vector potential in $2 \mathrm{D}$.

heating term in the energy equation. In the context of the momentum equation, the straightforward use of the vector potential term yields a second order operator $\mathbf{J} \times \mathbf{B}=\left(\frac{1}{\mu_{0}} \nabla \times \nabla \times \mathbf{A}\right) \times(\nabla \times \mathbf{A})$ that is difficult to integrate by parts to sufficiently reduce the order of the weak form operator. To avoid this difficulty, an alternate divergence form, assuming $\nabla \cdot \mathbf{B}=0$, is used to compute the Lorentz force as

$$
\mathbf{J} \times \mathbf{B}=\nabla \cdot\left[\frac{1}{\mu_{0}} \mathbf{B} \otimes \mathbf{B}-\frac{1}{2 \mu_{0}}\|\mathbf{B}\|^{2} \mathbf{I}\right] .
$$

In regards to the computation of the Joule heating source term in the energy Equation (3), a reformulation of this term appears not to be possible to avoid the requirement to compute a second derivative approximation for the current. Presently, this term is approximated by computing the curl of a field, $\hat{\mathbf{B}}$ that is obtained from an $L_{2}$ projection of the piecewise discontinuous approximation obtained from $\mathbf{B}=\nabla \times \mathbf{A}$.

\section{A Stabilized Finite Element Formulation for 2D Resistive MHD}

\subsection{Brief Overview of Stabilized Equations}

Table 1 presents the governing balance equations for momentum, total mass, thermal energy and the vector potential in residual form. These equations are currently solved in convected form and have been reduced to a low Mach number approximation form $[57,58]$ with an energy equation that is solved in terms of temperature. The low Mach number equations are closed by an equation of state of the form $P_{0}=\tilde{P}(T, \rho)$ that allows variation of density with temperature, $T$, and a global thermodynamic system pressure, $P_{0}$. The physical and transport properties are assumed to be functions of the local temperature, $T$, and density, $\rho$. This approach is similar to the formulation we have used in the context of modeling transport / reaction systems that are mixtures of ideal reacting gases in low speed chemical reactors [59-62]. In the illustrative numerical examples considered in this study, the density variation is modeled as incompressible and Boussinesq type fluids. However, the formulation as presented would be applicable to the more general low Mach number formulation.

The continuous PDE problem, defined by the low Mach number resistive MHD equations in Table 1, is approximated by a stabilized FE formulation. This formulation allows for stable equal-order velocitypressure interpolation and provides for convection stabilization as described below. In the case of a 
mixed Galerkin FE formulation of the momentum-continuity equations of the Navier-Stokes part of the MHD system, there is a stability requirement that the discrete spaces satisfy the LadyzhenskayaBabuska-Brezzi (LBB) condition; see e.g. [38] or [39]. This condition prevents the use of equal order finite element spaces, defined with respect to the same partition of the computational domain in finite elements. Linearization of the mixed nonlinear equations also leads to indefinite linear systems that are more difficult to solve by iterative methods. An additional difficulty is that the mixed Galerkin formulation is prone to instabilities for highly convected flows, even if the LBB condition is satisfied by the finite element spaces.

Consistently stabilized ${ }^{4}$ finite element methods for Navier-Stokes address these issues by using a combination of properly weighted residuals of the governing balance equations to simultaneously relax the incompressibility constraint and add streamline-diffusion, and sometimes nonlinear-discontinuitycapturing type operators, to the weak equations to limit oscillations in highly convected flows [46-50]. A significant added advantage of stabilization is that the linearized problems are real positive definite and therefore performance of iterative solvers can be improved [40].

The specific stabilized FE formulations employed in this study are shown in Table 2 . The stabilization parameters (the $\tau$ 's) are based on the formulations of Hughes and Mallet [63], Shakib [52], Hughes [64], and Tezduyar [65] for Navier-Stokes with an adaptation of the stabilized formulation of Codina and Hernandez-Silva [43] for a resistive MHD system. The definition of the stabilization parameters are provided in Table 3 for momentum, thermal energy, and the vector potential. The multidimensional effect of convection is incorporated into the stability parameters by the use of the contravariant metric tensor, $\mathbf{G}_{c}$ (Eqn (12)), of the transformation from local element coordinates $\left\{\zeta_{\alpha}\right\}$ to physical coordinates $\left\{x_{i}\right\}$. Shakib [52] considers the one dimensional limiting case of this multidimensional definition for the advection-diffusion equation and presents a comparison with the original SUPG technique,

$$
\left[\mathbf{G}_{c}\right]^{i j}=\frac{\partial \zeta_{\alpha}}{\partial x_{i}} \frac{\partial \zeta_{\alpha}}{\partial x_{j}} .
$$

Finally, it should be noted that this formulation, as presented, has some limitations. First this formulation is a simplification of proposed formulations for multiple advection-diffusion type equations that also couple the various equations in the definition of the least squares operators [63]. In addition there is no stabilization contribution for strong source terms and there is no nonlinear discontinuity capturing term present (see e.g.[63,66-68]). While we have experimented with these terms, they are not used in the results presented in this study.

\subsection{Brief Overview of Discrete Systems of Equations}

To give context to the discussion of solution methods and linear algebra, a brief discussion of the structure of the equations that result from the FE discretization of the weak form of the resistive MHD equations is presented. In this discussion, the Newtonian stress tensor is expanded to include the hydrodynamic pressure, $P$, and the viscous stress tensor term, $\boldsymbol{\Pi}$. The resulting stabilized from of the total mass residual equation in expanded form is given by

$$
\begin{gathered}
F_{P}=\int_{\Omega} \Phi\left[\frac{\partial \rho}{\partial t}+\nabla \cdot(\rho \mathbf{v})\right] d \Omega+ \\
\sum_{e} \int_{\Omega_{e}} \rho \hat{\tau}_{m} \nabla \Phi \cdot\left[\rho \frac{\partial \mathbf{v}}{\partial t}+\rho(\mathbf{v} \cdot \nabla \mathbf{v})+\nabla \cdot\left(-\frac{1}{\mu_{0}} \mathbf{B} \otimes \mathbf{B}-\mathbf{\Pi}+\left(P+\frac{1}{2 \mu_{0}}\|\mathbf{B}\|^{2}\right) \mathbf{I}\right)\right] d \Omega .
\end{gathered}
$$

$\overline{4}$ Consistent in the sense that the exact solution to the PDE equation satisfies the weak form residual equations 
Table 2

\begin{tabular}{|l|c|}
\hline Momentum & $\mathbf{F}_{m, i}=\int_{\Omega} \Phi \mathbf{R}_{m, i} d \Omega+\sum_{e} \int_{\Omega_{e}} \rho \hat{\tau}_{m}(\mathbf{v} \cdot \nabla \Phi) \mathbf{R}_{m, i} d \Omega$ \\
\hline Total Mass & $F_{P}=\int_{\Omega} \Phi R_{P} d \Omega+\sum_{e} \int_{\Omega_{e}} \rho \hat{\tau}_{m} \nabla \Phi \cdot \mathbf{R}_{m} d \Omega$ \\
\hline $\begin{array}{l}\text { Thermal } \\
\text { Energy }\end{array}$ & $F_{T}=\int_{\Omega} \Phi R_{T} d \Omega+\sum_{e} \int_{\Omega_{e}} \rho \hat{C}_{p} \hat{\tau}_{T}(\mathbf{v} \cdot \nabla \Phi) R_{T} d \Omega$ \\
\hline $\begin{array}{l}\text { Z- } \\
\text { component } \\
\text { Vector } \\
\text { Potential }\end{array}$ & $F_{A_{z}}=\int_{\Omega} \Phi R_{A_{z}} d \Omega+\sum_{e} \int_{\Omega_{e}} \hat{\tau}_{A_{z}}(\mathbf{v} \cdot \nabla \Phi) R_{A_{z}} d \Omega$ \\
\hline
\end{tabular}

Stabilized finite element formulation of transport/reaction PDEs, where the residual equations $R_{i}$ are presented in Table 1 and the stabilization parameters $\hat{\tau}_{i}$ are defined in Table 3.

\begin{tabular}{|l|c|}
\hline Momentum & $\hat{\tau}_{m}=\left[\left(\frac{2 \rho}{\Delta t}\right)^{2}+\rho^{2} \mathbf{v} \mathbf{G}_{c} \mathbf{v}+C_{1}^{2} \mu^{2}\left\|\mathbf{G}_{c}\right\|+C_{2}^{2}\|B\| \sqrt{\left\|\mathbf{G}_{c}\right\|}\right]^{-\frac{1}{2}}$ \\
\hline $\begin{array}{l}\text { Thermal } \\
\text { Energy }\end{array}$ & $\hat{\tau}_{T}=\left[\left(\frac{2 \rho C_{p}}{\Delta t}\right)^{2}+\left(\rho C_{p}\right)^{2} \mathbf{v} \mathbf{G}_{c} \mathbf{v}+C_{1}^{2} \lambda^{2}\left\|\mathbf{G}_{c}\right\|\right]^{-\frac{1}{2}}$ \\
$\begin{array}{l}\text { Z- } \\
\text { component } \\
\text { Vector } \\
\text { Potential }\end{array}$ & $\tau_{A_{z}}=\left[\left(\frac{2}{\Delta t}\right)^{2}+\mathbf{v G}_{c} \mathbf{v}+C_{1}^{2} \eta^{2}\left\|\mathbf{G}_{c}\right\|\right]^{-\frac{1}{2}}$ \\
\hline
\end{tabular}

Table 3

Definition of stabilization parameters used in stabilized equations, which use the contravarient metric tensor $\mathbf{G}_{c}$ (Eqn (12)) to define an element-level streamwise length scale.

This expansion includes the weak form of a Laplacian operator acting on pressure,

$$
L=\sum_{e} \int_{\Omega_{e}} \rho \hat{\tau}_{m} \nabla \Phi \cdot \nabla P d \Omega
$$

which is produced by the stabilized formulation of the total mass conservation equation.

Finite element (FE) discretization of the stabilized equations gives rise to a system of coupled, nonlinear, non-symmetric algebraic equations, the numerical solution of which can be very challenging. These equations are linearized using an inexact form of Newton's method as described in Section 4.2. A formal block matrix representation of these discrete linearized equations is given by

$$
\left[\begin{array}{cc}
\mathbf{K} & \mathbf{G} \\
\mathbf{D} \Gamma & \mathbf{L}
\end{array}\right]\left[\begin{array}{c}
\mathbf{v}^{\prime} \\
\mathbf{P}^{\prime}
\end{array}\right]=-\left[\begin{array}{l}
\mathbf{F}_{\mathbf{v}} \\
\mathbf{F}_{\mathbf{P}}
\end{array}\right]
$$

where the block diagonal contribution of the stabilization procedure has been highlighted by a specific ordering. In this representation, the vector, $\mathbf{v}^{\prime}$, contains the Newton updates to the nodal solution variables, $\left(\mathbf{v}, \mathbf{T}, \mathbf{A}_{\mathbf{z}}\right)$, with the exception of the nodal pressures, $\mathbf{P}^{\prime}$. The block matrix, $\mathbf{K}$, corresponds to the combined discrete transient, convection, diffusion and stress terms acting on the unknowns $\mathbf{v}^{\prime}$; the matrix, $\mathbf{G}$, corresponds to the discrete gradient operator; $\mathbf{D}$, the divergence operator; the diagonal matrix, $\boldsymbol{\Gamma}$, results from the group FE expansion of the density and velocity; and the matrix, $\mathbf{L}$, corresponds to the discrete "pressure Laplacian" operator discussed above. The vectors $\mathbf{F}_{\mathbf{v}}$ and 
$\mathbf{F}_{\mathbf{P}}$ contain the right hand side residuals for Newton's method. Our current linear algebra solution procedure uses a specific ordering of the unknowns locally at each FE node with each degree of freedom ordered consecutively. A single coupled matrix problem, $\mathbf{J}_{\mathbf{s}}=\mathbf{- F}$, is solved at each Newton step with sophisticated algebraic domain decomposition and multilevel preconditioned Krylov methods as described in Section 4.3.

The existence of the well conditioned nonzero matrix, $\mathbf{L}$, in the stabilized FE discretization of the equations allows the solution of the linear systems with a number of algebraic and domain decomposition type preconditioners $[69,70]$. This is in contrast to other formulations, such as Galerkin methods using mixed interpolation, that produce a zero block on the total mass continuity diagonal. The difficulty of producing robust and efficient preconditioners for the Galerkin formulation has motivated the use of many different types of solution methods. A number of these use two-level iteration schemes, penalty methods, pseudo-compressibility techniques or decoupled/segregated solvers (e.g. [71,72,?]). A detailed presentation of the characteristics of current solution methods is far beyond the scope of this brief overview. However, the intent of our method of fully-coupling the transport PDEs in the nonlinear solver is to preserve the inherently strong coupling of the physics with the goal to produce a more robust solution methodology. Preservation of this strong coupling, however, places a significant burden on the linear solution procedure to solve the fully coupled algebraic systems.

\section{Fully-implicit Fully-coupled Solution by Parallel Newton-Krylov Methods}

\subsection{Fully-implicit Time Integration and Direct to Steady-state Solutions}

As described above, the resistive MHD systems can exhibit a very large range of interacting times scales induced by the multi-physics character of these equations. For multiple-time-scale systems, fullyimplicit methods can be an attractive choice that can often provide unconditionally-stable time integration techniques. These methods can be designed with various stability properties (e.g. A-, L-stability) that allow robust integration of multiple timescale systems without the requirement to resolve the stiff modes of the system that are not of interest and do not control the accuracy of time integration $[2,3]$. The stability of these methods, however, comes at a price, as these techniques generate very large, coupled and highly nonlinear sparse systems of equations that must be solved at each time step.

The time integration methods used in this initial study of fully-implicit methods for resistive MHD include the first-order A \& L-stable backward Euler Method and the second-order A-stable implicit midpoint rule $[2,3]$. For notational purposes the time dependence of the governing equations and the dependence on the physical parameters of the system are made explicit as follows. Formally we represent this time dependent nonlinear system of coupled equations in Table 2 as

$$
\mathbf{F}(\dot{\mathbf{U}}, \mathbf{U}, \mathbf{p})=\mathbf{0}
$$

where $\mathbf{F}$, is the vector of residuals for the stabilized FE equations, $\mathbf{U}$, is the vector of unknowns, and the list of parameters on which the system depends is denoted by $\mathbf{p}$.

The first-order backward Euler method (BE) solves the system:

$$
\mathbf{F}\left(\dot{\mathbf{U}}^{n+1}, \mathbf{U}^{n+1}, \mathbf{p}\right)=\mathbf{0}
$$

with

$$
\dot{\mathbf{U}}^{n+1} \equiv \frac{\mathbf{U}^{n+1}-\mathbf{U}^{n}}{\Delta t}
$$


The second-order Mid-point rule (Mid Point) implicit integrator solves the system:

$$
\mathbf{F}\left(\dot{\mathbf{U}}^{n+\frac{1}{2}}, \mathbf{U}^{n+\frac{1}{2}}, \mathbf{p}\right)=\mathbf{0},
$$

with

$$
\dot{\mathbf{U}}^{n+\frac{1}{2}} \equiv \frac{\mathbf{U}^{n+1}-\mathbf{U}^{n}}{\Delta t} ; \quad \mathbf{U}^{n+\frac{1}{2}}=\frac{1}{2}\left(\mathbf{U}^{n+1}+\mathbf{U}^{n}\right)
$$

In the case of a direct-to-steady-state solution process to find a solution, $\overline{\mathbf{U}}$, of Eqn (16) for the parameter values, $\overline{\mathbf{p}}$, the nonlinear systems of equations becomes:

$$
\mathbf{F}(0, \overline{\mathbf{U}}, \overline{\mathbf{p}})=\mathbf{0} .
$$

The result of a direct-to-steady-state and fully-impliciit solution technique is the development of very large-scale, coupled highly nonlinear system(s) that must be solved. Therefore, these techniques place a heavy burden on the nonlinear and linear solvers and require robust, scalable and efficient nonlinear solution methods such as the preconditioned Newton-Krylov methods discussed in Section 4.2 and 4.3 .

\subsection{Inexact Newton Methods}

As described above, the stabilized FE formulation of the governing transient and steady-state resistive MHD equations form a strongly coupled nonlinear system of equations (Eqn (16) and Eqn (21)). To provide robust and efficient solution of these challenging systems, fully-coupled Newton-based iterative nonlinear solvers [73] are employed. These solvers can exhibit quadratic convergence rates independently of the problem size for sufficiently robust linear solvers. A Newton-Krylov method [74,31] is an implementation of Newton's method in which a Krylov iterative solution technique is used to approximately solve the linear systems that are generated at each step of Newton's method. Specifically, to solve the nonlinear system $\mathbf{F}(\mathbf{U})=\mathbf{0}$, we seek a zero of $\mathbf{F}: R^{N} \rightarrow R^{N}$ where $\mathbf{U} \in R^{N}$ is a current approximate solution. The Krylov iterative solver is applied to determine an approximate solution of the Newton equation

$$
\mathbf{J}_{k} \mathbf{s}_{k+1}=-\mathbf{F}_{k},
$$

where $\mathbf{J}_{k}$ is the Jacobian matrix, and $\mathbf{F}_{k}$ is the nonlinear residual evaluated at the previous Newton step solution $\mathbf{U}_{k}$. The solution for the Newton direction vector, $\mathbf{s}_{k+1}$, is used to update the previous solution in the sequence as $\mathbf{U}_{k+1}=\mathbf{U}_{k}+\theta \mathbf{s}_{k+1}$, where $\theta$ is a step length reduction or backtracking parameter. Back-tracking algorithms are techniques for improving the robustness of the nonlinear solver, and work by scaling the Newton correction vector by a parameter, $\theta$, as needed to ensure that the nonlinear residual has been reduced adequately before the step is accepted [73,75].

For efficiency, an inexact Newton method [76-78] is usually employed, whereby one approximately solves Eqn (22) by choosing a forcing term $\eta_{k+1}$ and stopping the Krylov iteration when the inexact Newton condition is satisfied, namely:

$$
\left\|\mathbf{F}_{k}+\mathbf{J}_{k} \mathbf{s}_{k+1}\right\| \leq \eta_{k+1}\left\|\mathbf{F}_{k}\right\|
$$

In this context an inexact Newton method uses nonlinear residual information to determine the accuracy $\eta_{k+1}$, to which the sequence of linear subproblems are solved. Specific choices for $\eta_{k+1}$, in the inexact Newton scheme and a more thorough numerical evaluation of these methods can be found in $[77,79,80]$. In the implementation of Newton's method presented in this study we use a constant value of the $\eta_{k+1}$ parameter to focus on the performance of the preconditioners. In addition, the Jacobian 
matrix, $\mathbf{J}$ that is used for the Jacobian vector products in the Krylov solvers, and as the basis for computing the preconditioners described in Section 4.3, is developed from automatic differentiation (AD) techniques. These methods are applied to the programmed functions representing the weak form residuals outlined in Table 2 by employing the SACADO package from the Trilinos framework [81].

Finally by developing a direct-to-steady-state solution capability based on Newton-Krylov type techniques, additional advanced solution methods such as parameter continuation, bifurcation tracking and linear stability analysis algorihtms, can be effectively developed to analyze complex nonlinear solution spaces of interest in the design and operation of physical systems of scientific and technological interest (e.g. [82-86]).

\subsection{Schwarz Domain Decomposition and Multilevel Preconditioners}

For the considered class of problems, convergence is not achieved without preconditioning due to ill-conditioning in the underlying matrix equations [87]. This paper considers one-level Schwarz preconditioners where the basic idea is to decompose the computational domain $\Omega$ into overlapping subdomains $\Omega_{i}$ and then assign each subdomain to a different processor [33,34]. One application consists of solving on subdomains and then combining these local solutions to construct a global approximation throughout $\Omega$. The $i^{\text {th }}$ subdomain problem is usually defined by enforcing homogeneous Dirichlet boundary conditions on the subdomain boundary, $\partial \Omega_{i}$. In the minimal overlap case, the algebraic Schwarz method corresponds to block Jacobi where each block contains all degrees of freedom (DOFs) residing within a given subdomain. Convergence is typically improved by introducing overlap which can be done recursively. Specifically, the $j^{\text {th }}$ block within a $(k+1)$-overlap version contains DOFs corresponding to nonzero columns within the matrix rows used to define the $j^{\text {th }}$ block in a $k$-overlap version. Solutions in overlap regions are usually combined by either averaging or simply taking the solution from only one subdomain within different overlap subregions, i.e. ignoring solutions from all but one subdomain within a subregion.

Zoltan is used to automatically partition the computational domain over processors which implicitly defines the subdomains [88]. Incomplete factorization, $I L U(k)$, is employed to approximate the solution of the local Dirichlet problems and avoid the large cost of direct factorization $[87,89]$. Typically, $k$ is chosen so that the incomplete factorization requires a bit more than twice as much storage as the original matrix. Ifpack performs these incomplete factorizations [90] and Aztec [91] implements the Krylov methods, e.g. GMRES. We note that the one-level preconditioner is black-box in that the overlapping subdomain matrices are constructed completely algebraically.

A possible drawback of one-level Schwarz is its locality. One application mixes information between neighboring sub-domains. This implies that many repeated applications are required to combine information across the entire domain. Thus, as the number of subdomains increases, the convergence rate deteriorates for standard elliptic problems due to this lack of global coupling [33]. The convergence rate also deteriorates as the number of unknowns per subdomain increases when ILU $(k)$ is used for a subdomain solver. To remedy this, coarse levels can be introduced to approximate global coupling [92,70]. The use of a coarse mesh to accelerate the convergence of a one-level Schwarz preconditioner is similar to multigrid methods that use a sequence of coarser meshes [93,94]. Typically, more than two levels are employed in a multigrid approach.

In this paper, only algebraically generated coarse levels are considered. These are significantly easier to implement and integrate with a complicated unstructured simulation as opposed to geometric coarse grids $[92,70,84]$. In particular, there is no need to represent complex geometric features on all levels, e.g. faces, edges, and corners to define the domain boundary. There is also no requirement to interact with the simulation's geometric data structures. Most algebraic multigrid methods (AMG) associate a 
graph with the matrix system being solved. Graph vertices correspond to matrix rows for scalar PDEs while for PDE systems it is natural to associated one vertex with each nodal block of unknowns, e.g. velocities and pressures at a particular grid point. A graph edge exists between vertex $i$ and $j$ if there is a nonzero in the block matrix which couples $i$ 's rows with $j$ 's columns or $j$ 's rows with $i$ 's columns. In some situations it may be advantageous to omit edges if all entries within the coupling block are small [95]. Once defined, the graph can be coarsened followed by the construction of grid transfer operators to move solutions between coarse and fine representations. The fine grid discretization can now be projected to coarser levels in a Galerkin or Petrov-Galerkin fashion using a triple matrix product. The AMG method is fully specified when the smoothers or approximate solvers on each level are fixed.

Perhaps the most well-known AMG technique is the classical approach of Ruge-Stüben [96,95] where a subset of fine mesh vertices are used to define the next coarser mesh. In this paper, an alternative based on smoothed aggregation is employed [97,98]. Fine mesh vertices are grouped into aggregates so that each aggregate effectively represents a coarse mesh vertex. Standard smoothed aggregation typically utilizes aggregates with about 30 nodes each for three dimensional isotropic problems. In this study, METIS and ParMETIS [99] define our aggregates. These packages subdivide the matrix graph so that each partition has no more nodes than a user supplied parameter and that each partition is somewhat spherically shaped. We orient METIS and ParMETIS so that they generate somewhat larger aggregates than those typically used in standard smoothed aggregation. This aggressive coarsening significantly reduces the number of unknowns between consecutive levels. This generally limits the total number of levels $(\leq 5)$ which we find better suited for parallel computations [35,100]. Addtionally, larger aggregates are consistent with using Schwarz/ILU $k$ ) which in the multigrid context corresponds to a somewhat heavyweight smoother (compared to Gauss-Seidel often used in standard multigrid). That is, one can coarsen more aggressively when a more substantial smoother is employed. The same $I L U(k)$ algorithm is used as a smoother on each level and on the coarsest level the KLU [101,102] sparse direct solver is employed.

Once the coarse mesh is determined, an initial grid transfer is constructed corresponding to piecewise constant interpolation. ${ }^{5}$ The grid transfer matrix, $P$, contains only zeros and ones. In the scalar PDE case, $P_{i j}$ equals one only if the $i^{\text {th }}$ fine grid point has been assigned to the $j^{\text {th }}$ aggregate. Within a PDE system, the grid transfer is a block system with an identity matrix for the $(i, j)^{t h}$ block if the $i^{\text {th }}$ fine grid point has been assigned to the $j^{\text {th }}$ aggregate. This initial grid transfer can then be improved by smoothing the corresponding basis functions. In the symmetric elliptic case, this is well understood and essentially corresponds to damped Jacobi, e.g. $\hat{p}_{j}=\left(I-\omega D^{-1} A\right) p_{j}$ where $p_{j}$ is the $j^{\text {th }}$ basis function in the intial grid transfer, $\hat{p}_{j}$ is the new basis function, $\omega$ is a damping parameter, $A$ is the discretization matrix, and $D$ is the diagonal of $A$. The situation is less clear in the nonsymmetric case. A Petrov-Galerkin smoothed aggregation algorithm is given in [103] which smooths separately the restriction and prolongation operators and also replaces the single damping parameter with a diagonal damping matrix. The convergence rate of standard smoothed aggregation AMG does not deteriorate as the mesh is refined for symmetric elliptic problems. This has been shown both theoretically and computationally $[97,98]$. The Petrov-Galerkin version has a much weaker theoretical base though computationally it generally converges better than the standard method on highly convective problems and also exhibits scalable convergence rates (no deterioration with refinement) on a large number of highly convective problems [103]. In this study, we consider both the Petrov-Galerkin scheme as well as the simplier scheme based on the initial grid transfer corresponding to piecewise-constants [35,104].

Multilevel implementation is provided by ML $[92,105]$. ML has been employed successfully in a num-

\footnotetext{
5 In more general settings the initial transfer might exactly interpolate more complex functions such as rigid body modes with piecewise versions of these functions.
} 
ber of applications that include stabilized FE discretizations of Navier-Stokes and transport-reaction type systems [106,84,107], as well as drift-diffusion systems for semi-conductor modeling [108]. Aztec, Ifpack, KLU, ML, KLU, and Zoltan are available through the Trilinos framework [81].

\section{Results and Discussion}

\subsection{Representative Verification and Order of Accuracy Results}

In this section detailed numerical order-of-accuracy results are presented for a set of $2 \mathrm{D}$ resistive MHD problems that admit analytic solutions that can be used for verification.

\subsubsection{Flux Expulsion Problem}

This problem has an analytical solution that is used as a verification problem in the evaluation of numerical methods for resistive MHD [109,42]. It consists of solving for the vector potential solution with a prescribed discontinuous velocity field. The problem consists of a rotating infinitely long cylindrical conductor immersed in a conducting fluid with the solid body rotational velocity field $\mathbf{v}=\left(-\omega_{0} y, \omega_{0} x, 0\right)$ in the cylinder and $\mathbf{v}=\mathbf{0}$ outside the cylinder. The dependent variable is $\mathbf{A}=\left(0,0, A_{z}\right)$. The geometry is taken as a cylinder of radius, $R_{0}$ within a computational domain $\Omega=[-2,2] \times[-1,1]$ with a linear varying potential field $A_{z}=B_{0} y$ that produces a uniform magnetic flux $\mathbf{B}=\left(B_{0}, 0,0\right)$. The analytic solution is given by

$$
A_{z}=\mathfrak{I m}\left[B_{0} f(r) e^{i \theta}\right]
$$

where

$$
\begin{array}{ll}
f(r)=r+\frac{C}{r}, & r>r_{0} \\
f(r)=D J_{1}(p r), & r \leq r_{0}
\end{array}
$$

with

$$
\begin{array}{rlrl}
p= & \frac{(1-i) k_{0}}{\sqrt{2}}, & k_{0}=\frac{\sqrt{R e_{m}}}{r_{0}} \\
C=\frac{r_{0}\left[2 J_{1}\left(p r_{0}\right)-p r_{0} J_{0}\left(p r_{0}\right)\right]}{p J_{0}\left(p r_{0}\right)}, & D=\frac{2}{p J_{0}\left(p r_{0}\right)}
\end{array}
$$
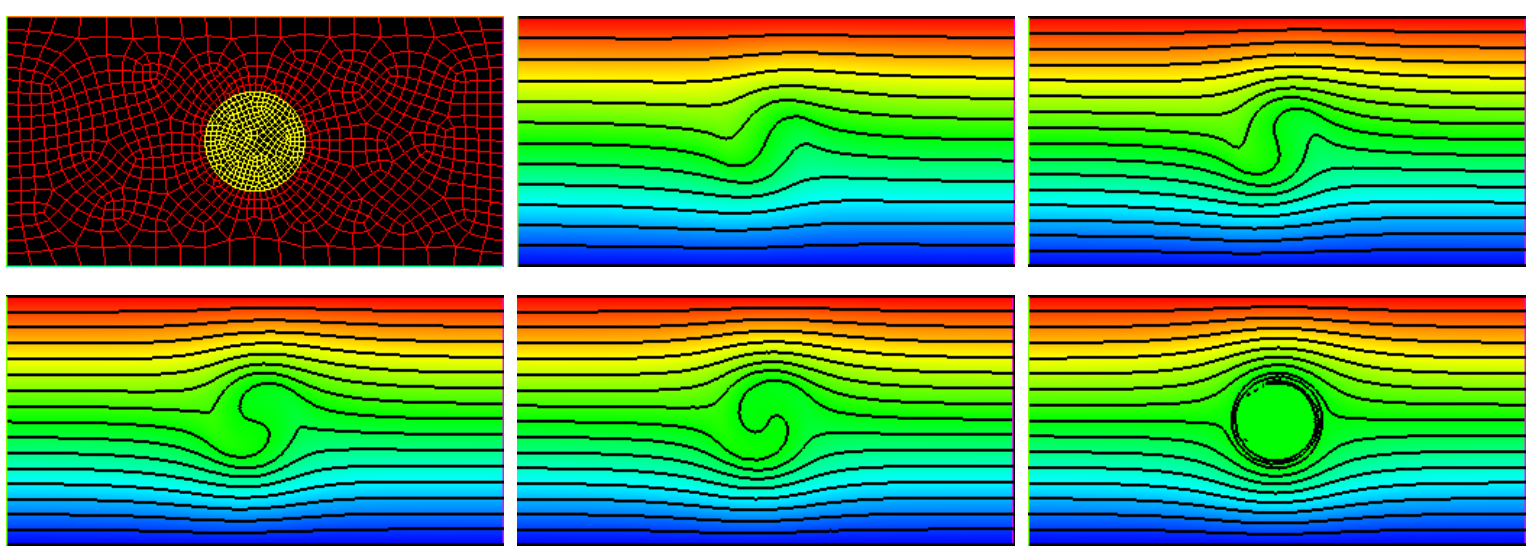

Fig. 1. Flux Expulsion verification problem. The top left figure shows a coarse computational unstructured mesh $\Omega=[-1,1] \times\left[-\frac{1}{2}, \frac{1}{2}\right]$. The remaining figures show contour plots of numerical solution of $A_{z}$ for $R e_{m}=6,12,24,48,12288$ from the top center image to the lower right image. 

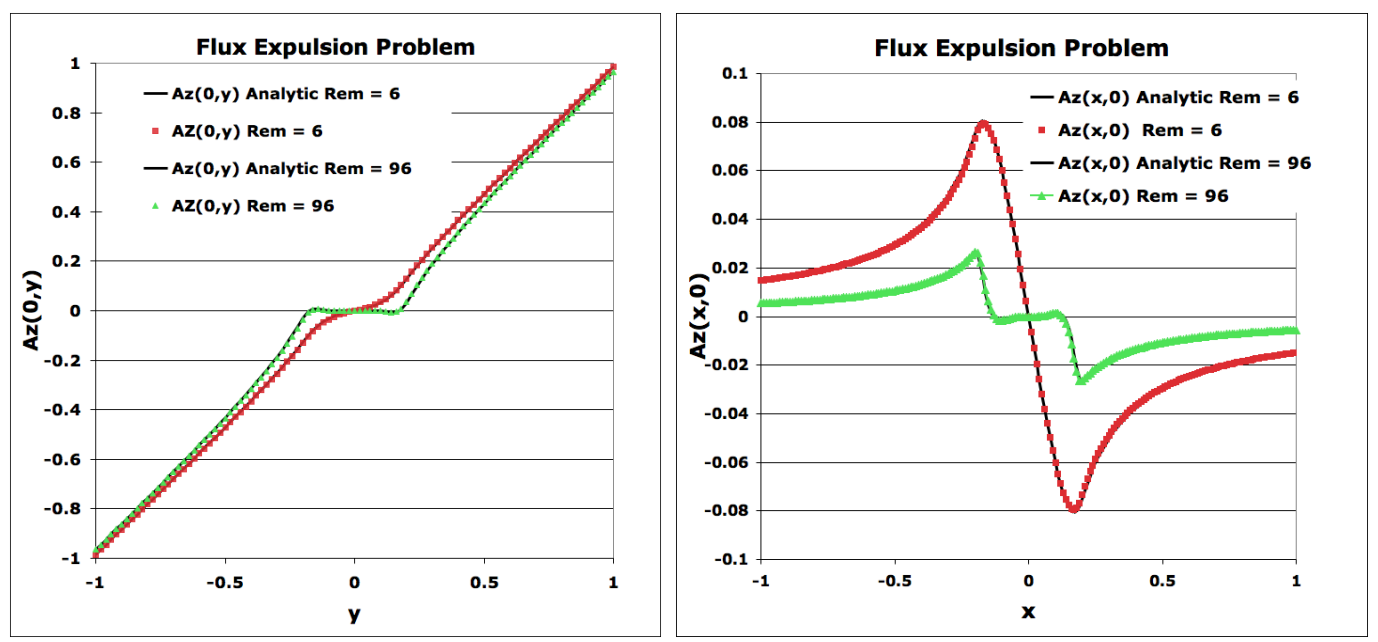

Fig. 2. Flux Expulsion verification problem. Figures show profiles of analytic solution of $A_{z}(y, 0)($ left $)$ and $A_{z}(x, 0)$ (right) along with numerically computed solutions from the vector potential solution in $\Omega=[-2,2] \times[-1,1]$ with $20 \mathrm{~K}$ unstructured quad elements. The red squares are for $\operatorname{Re}_{m}=6$ and the green triangles are for the more highly convected case of $R e_{m}=96$, the black line is the analytic solution due to Moffat [109].
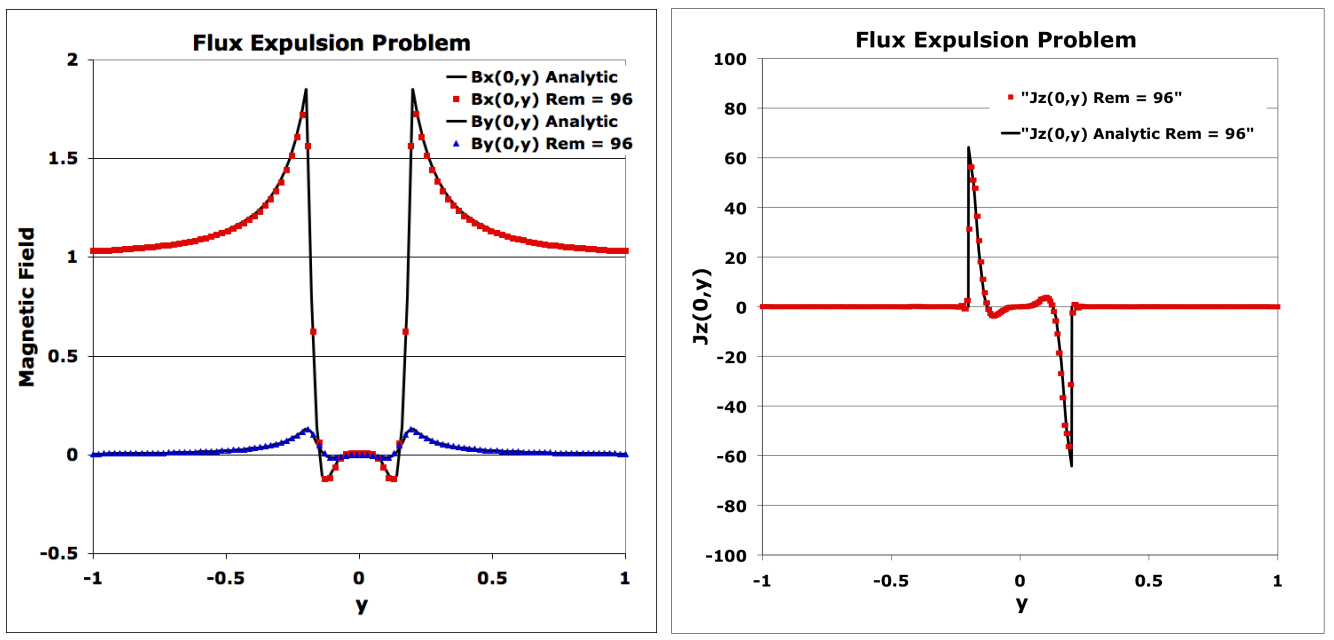

Fig. 3. Flux Expulsion verification problem. Figures show profiles of analytic solution of $B_{x}(0, y)$ and $B_{y}(0, y)$ (left) along with $J_{z}(0, y)$ (right) along with numerically computed solutions from the vector potential solution in $\Omega=[-2,2] \times[-1,1]$ with $20 \mathrm{~K}$ unstructured quad elements. The black line is the analytic solution due to Moffat [109].

The computational solution is produced on an unstructured mesh, a coarse example of which is presented in Figure 1. As the rotational rate is increase in the conductor, the linear $A_{z}$ (uniform $B_{x}$ ) field is distorted. At high rotation rates the field is expelled from the cylinder and the effects of diffusion modify the flux field in the region about the rotating conductor. Figure 1 shows iso-lines of $A_{z}$ as a function of the magnetic Reynolds number, $R e_{m}=U R_{0} / \eta$. A comparison of the analytical solution with the numerical solution is provided in Figure 2 where $A_{z}(x, 0)$ and $A_{z}(0, y)$ are shown in a region about the conductor. Figure 3 presents the results for the post-processed variables $B_{x}(0, y), B_{y}(0, y)$ and $J_{z}(0, y)$ computed on the $20 \mathrm{~K}$ element unstructured mesh. There has been no significant optimization of the mesh to resolve the highly localized induced magnetic field and plasma current internal layers near the rotating inner cylinder. The computed values show very good agreement with the analytic results of Moffat [109]. These comparisons indicate a very good agreement between the computed solutions 
and the exact analytical solution on a reasonably coarse unstructured mesh.

\subsubsection{A Modified Hartmann Flow Vector Potential Problem}

This problem has an analytical solution [110] that is often used as a verification problem in the evaluation of numerical methods for resistive MHD [37,111]. In the classical form, this is a 1D solution and the dependent variables are $(\mathbf{v}, P, \mathbf{B})$. The geometry is taken as a square box with $(x, y) \in[-L, L] \times$ $[-L, L]$ with an assumed pressure gradient that drives the flow of $\frac{\partial P}{\partial x}=-G_{0}$. The analytic asymptotic solution is of the form $\mathbf{B}=\left(B_{x}, B_{0}, 0\right)$ and $\mathbf{v}=\left(v_{x}, 0,0\right)$, where $B_{y}=B_{0}$ is an applied external magnetic field that retards the flow with the tension in the magnetic field lines. The solution is given by

$$
\begin{aligned}
v_{x} & =-\frac{\rho G_{0} H a}{\mu_{0} B_{0}^{2}}\left[\frac{\cosh (H a)-\cosh (y H a / L)}{\sinh (H a)}\right] \\
B_{x} & =-\frac{B_{0} R e_{m}}{H a}\left[\frac{\sinh (y H a / L)-(y / L) \sinh (H a)}{\cosh (H a)-1}\right]
\end{aligned}
$$

In the context of a vector potential formulation it can be shown that an analogous modified Hartmann problem can be defined that has $\nabla \times \mathbf{A}=\left(B_{x}, B_{0}, 0\right)$ with $\mathbf{A}=\left(0,0, A_{z}\right)$. The solution vector has the form, $\left(\mathbf{v}, P, A_{z}\right)$ and the solution for the vector potential is

$$
A_{z}=-B_{0} x-\frac{G_{0} y^{2}}{2 B_{0}}+\frac{G_{0}}{B_{0}}[H a \cosh (y H a) \operatorname{csch}(H a)]
$$

and must be sustained by an external electric field with

$$
E_{z}^{0}=\frac{G_{0}}{B_{0}}[H a \operatorname{coth}(H a)-1]
$$

In Figure 5, contour plots of the numerical solution for $v_{x}$ and $A_{z}$ are presented along with the post-processed quantity $B_{x}$. The $1 \mathrm{D}$ nature of $v_{x}$ and $B_{x}$ are evident along with the $2 \mathrm{D}$ variation of the vector potential $A_{z}$. In Figure 5 profiles from the computed numerical solution are compared with the analytic solution for $v_{x}$ and $B_{x}$ at $x=0$ for various values of $R e=U(2 L) / \nu=R e_{m}=U(2 L) / \eta$ and Hartmann number, $H a=B_{0}(2 L) / \sqrt{\mu_{0} \rho \mu \eta}$. This figure shows qualitatively excellent agreement to the analytical solution. Figure 6 shows a detailed spatial convergence study for a mesh with spacing $\Delta x=\Delta y$. The expected order of convergence for linear nodal elements in the dependent variables $v_{x}$ and $A_{z}$ is exhibited with super-convergence of the quantity $B_{x}$ computed by a lumped mass $L_{2}$ projection.
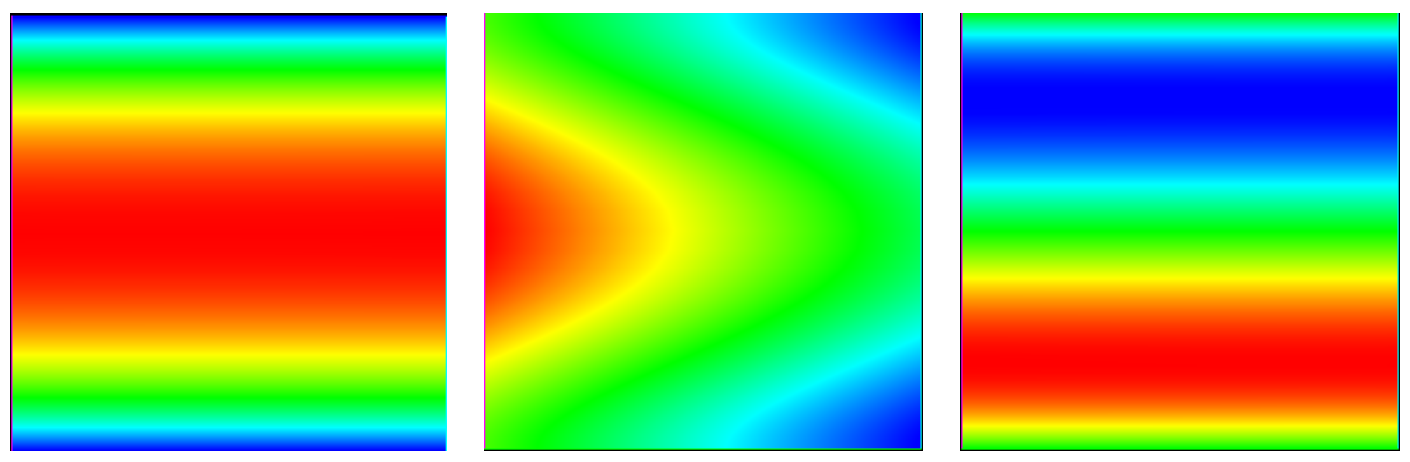

Fig. 4. Modified Hartmann flow verification problem, $R e=R e_{m}=2 V_{x}$. Figures show contour plots of numerical solution of $v_{x}$ (left), $A_{z}$ (center), and $B_{x}$ (right). 

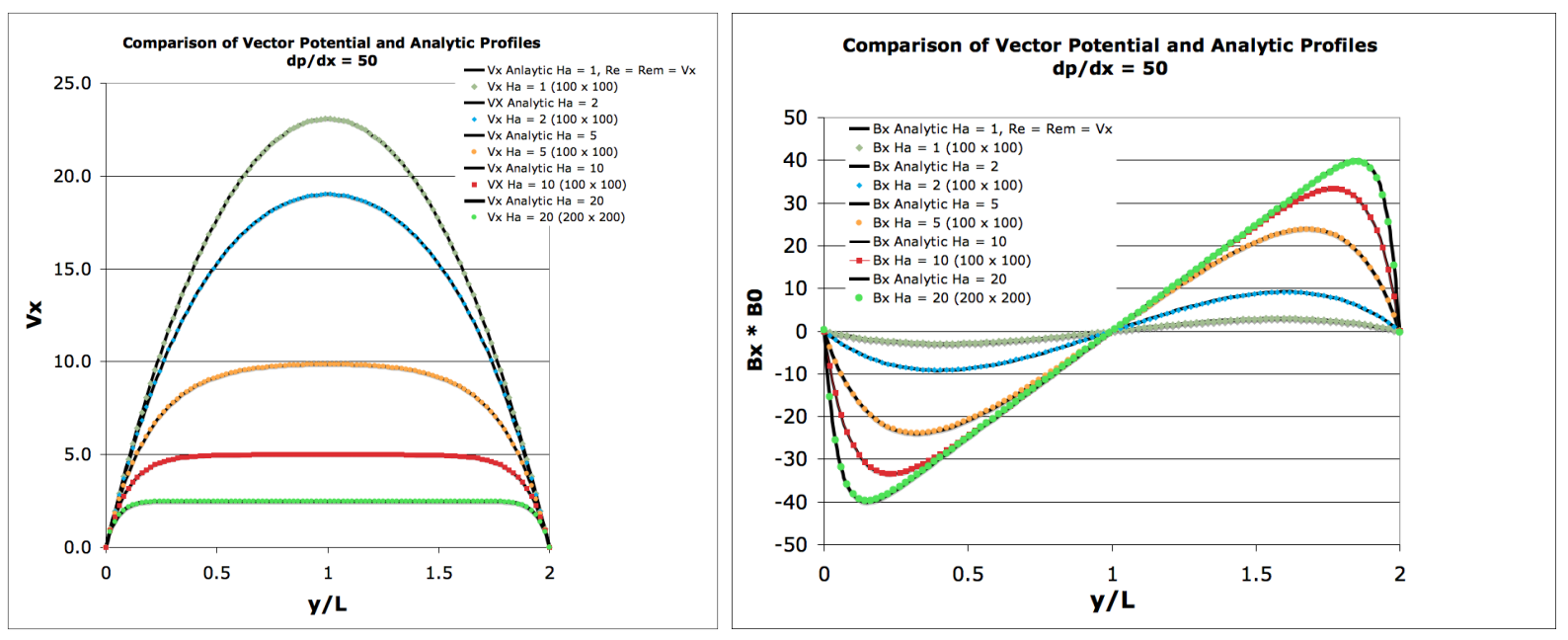

Fig. 5. Modified Hartmann flow verification problem, $R e=R e_{m}$. Figures show profiles of analytic solution of $v_{x}$ (left) and $B_{0} B_{x}$ (right) along with numerically computed solutions at $x=0.0$ from the vector potential solution.

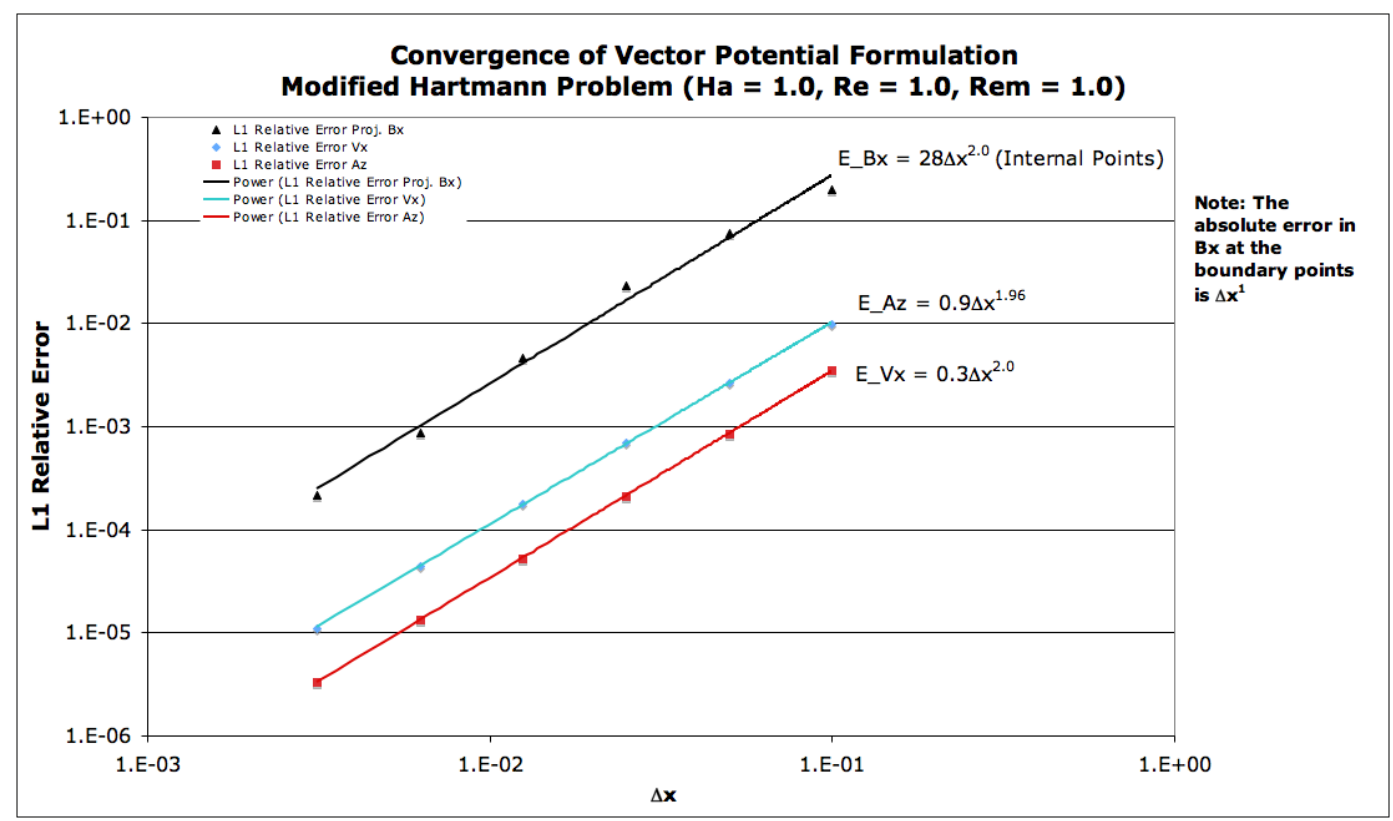

Fig. 6. Modified Hartmann flow verification problem, $R e=R e_{m}$. Figure shows convergence of the computed numerical solution for $v_{x}, A_{z}$ and the derived quantity $B_{x}$ from the vector potential solution.

As a final illustration with this type of MHD duct flow problem, a simple study of the effect of the convection stabilization by the SUPG-type terms for a strongly convected flow is presented. This simple prototype geometry is a rectangular channel with $(x, y) \in[0,5] \times[-1,1]$ and a very coarse mesh with size $10 \times 20$ is employed. The inlet has velocity $v_{x}(0, y)=V_{0}$ with $V_{0}=100,200,400$ with appropriate outlet Neumann conditions. An external perpendicular magnetic field is imposed by a linear $\operatorname{ramp}$ in $A_{z}$ with $A_{z}(0, y)=-5$ and $A_{z}(5, y)=5$. For high flow velocities the strongly enforced Dirichlet condition at $x=5$ produces a very sharp boundary layer. For cell based $R e=R e_{m}>1$ the Galerkin discretization can become unstable and produce oscillations. As expected the SUPG stabilization helps to control and localize these oscillations. Clearly from Figure 7 the nonphysical behavior of the Galerkin discretization is apparent. The stabilized method clearly helps to control the large-scale oscillations found in the Galerkin discretization. While there still appears to be some slight outflow effects seen in the velocity profile for the stabilized case, this most likely is due to the use of an inaccurate lumped mass projection 
to recover the $\mathbf{B}$ field. This technique has problems at boundaries and therefore produces a less accurate computation of the PDE residual and the $\mathbf{J} \times \mathbf{B}$ Lorentz force term. A consistent mass projection is being explored.
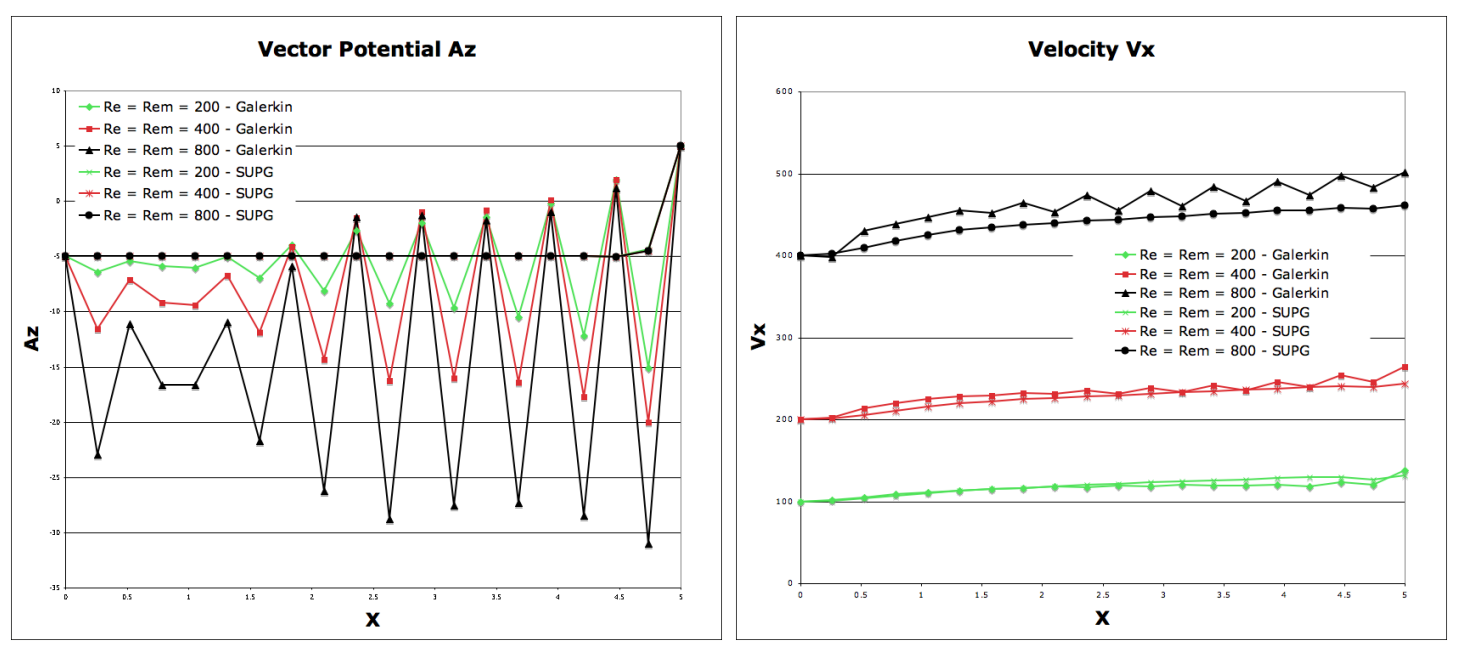

Fig. 7. Test of the SUPG-type stabilization of convective effects. Figures show profiles of $A_{z}$ (left) and $v_{x}$ (right). The Hartmann number for this case is 4 . Note that in the left figure the SUPG results are indistinguishable for all $R e_{m}$.

\subsubsection{MHD Rayleigh Flow and Alfven Wave Propagation Problem}

This problem has an analytical solution [110] that can used as a verification problem in the evaluation of numerical methods for transient resistive MHD [37]. In the classical form, this is a $1 \mathrm{D}$ transient problem that has an infinite plate bounding a conducting fluid in a semi-infinite domain. There is an externally applied magnetic field in the $y$-direction with magnitude $B_{0}$. The plate is initially at rest and then is suddenly set in motion with a velocity $U$. A viscous boundary layer flow is developed where the velocity profile is modified from the classical Rayleigh flow profile [112] by the existence of the magnetic field. As the velocity profile is developed, a self-induced magnetic field in the $x$-direction $B_{x}$ is developed as an Alfven wave with velocity $A_{0}=B_{0} / \sqrt{\mu_{0} \rho}$ propagates into the fluid. In the numerical solution the infinite half-space is approximated as a square box with $(x, y) \in[0,5] \times[0,5]$. For the case where the magnetic Prandtl number $P r_{m}=1$ the analytic asymptotic solution is of the form $\mathbf{B}=\left(B_{x}, B_{0}, 0\right)$ and $\mathbf{v}=\left(v_{x}, 0,0\right)$. The solution is given by

$$
\begin{aligned}
v_{x}=\frac{1}{4} U\left[e^{-\frac{A_{0} y}{d}}\left(1-\operatorname{erf}\left(\frac{y-A_{0} t}{2 \sqrt{d t}}\right)\right)-\operatorname{erf}\left(\frac{y-A_{0} t}{2 \sqrt{d t}}\right)\right] \\
\quad+\frac{1}{4} U\left[e^{\frac{A_{0} y}{d}}\left(1-\operatorname{erf}\left(\frac{A_{0} t+y}{2 \sqrt{d t}}\right)\right)-\operatorname{erf}\left(\frac{A_{0} t+y}{2 \sqrt{d t}}\right)+2\right] \\
B_{x}=-\frac{1}{4} e^{-\frac{A_{0} y}{d}}\left(-1+e^{\frac{A_{0} y}{d}}\right) U \sqrt{\mu \rho}\left(\operatorname{erfc}\left(\frac{y-A_{0} t}{2 \sqrt{d t}}\right)+e^{\frac{A_{0} y}{d}} \operatorname{erfc}\left(\frac{A_{0} t+y}{2 \sqrt{d t}}\right)\right)
\end{aligned}
$$

In the context of a vector potential formulation it can be shown that an analogous modified problem can be defined that has $\nabla \times \mathbf{A}=\left(B_{x}, B_{0}, 0\right)$ with $\mathbf{A}=\left(0,0, A_{z}\right)$. The solution vector has the form, 
$\left(\mathbf{v}, P, A_{z}\right)$ and the $2 \mathrm{D}$ solution for the vector potential is

$$
\begin{array}{rl}
A_{z}=-B_{0} & x+\frac{U \sqrt{d t} \sqrt{\mu \rho}}{2 \sqrt{\pi}}\left(e^{-\frac{\left(y-A_{0} t\right)^{2}}{4 d t}}-e^{-\frac{\left(y+A_{0} t\right)^{2}}{4 d t}}\right) \\
& +\frac{U}{4} \frac{\sqrt{\mu \rho}}{A_{0}}\left(d+A_{0}^{2} t\right)\left(\operatorname{erf}\left(\frac{A_{0} t-y}{2 \sqrt{d t}}\right)-\operatorname{erf}\left(\frac{A_{0} t+y}{2 \sqrt{d t}}\right)\right) \\
& -\frac{U}{4} \frac{\sqrt{\mu \rho}}{A_{0}} e^{\frac{-A_{0} y}{d}}\left(d+A_{0} e^{\frac{A_{0} y}{d}} y\right) \operatorname{erfc}\left(\frac{y-A_{0} t}{2 \sqrt{d t}}\right) \\
& -\frac{U}{4} \frac{\sqrt{\mu \rho}}{A_{0}}\left(d e^{\frac{A_{0} y}{d}}-A_{0} y\right) \operatorname{erfc}\left(\frac{A_{0} t+y}{2 \sqrt{d t}}\right)
\end{array}
$$

and must be sustained by an external electric field with $E_{z}^{0}=\frac{B_{0} U}{2}$.

The parameters in this problem are taken as in [37] to be $U=1.0, B_{0}=1.4494 e^{-4}, \rho=0.4 e^{-4}$, $\mu_{0}=1.256636 e^{-6}, \eta=1.256636 e^{-6}$, and $\mu=0.4 e^{-4}$ with $d=\eta / \mu_{0}=\mu / \rho=1$. Dimensionless parameters values should be shown here. The initial conditions and boundary conditions are defined by the analytical solution above. In Figures 8 and 9, line plots of the analytic and numerical solution for $v_{x}$, the post-processed quantity $B_{x}$, and $A_{z}$ are presented at various discrete times. In this plot the Hartmann layer in $v_{x}$ near the plate surface at $y=0$, and the plateau region influenced by the magnetic field is evident. These figures show qualitatively excellent agreement to the analytical solution. Figure 10 shows a detailed spatial and temporal order-of-accuracy study. The expected order of spatial order-of-accuracy for linear nodal elements in the dependent variables $v_{x}$ and $A_{z}$ is exhibited with super-convergence of the quantity $B_{x}$ computed by a lumped mass $L_{2}$ projection. This figure exhibits the expected order-of-accuracy for the first and second order temporal integration as well.
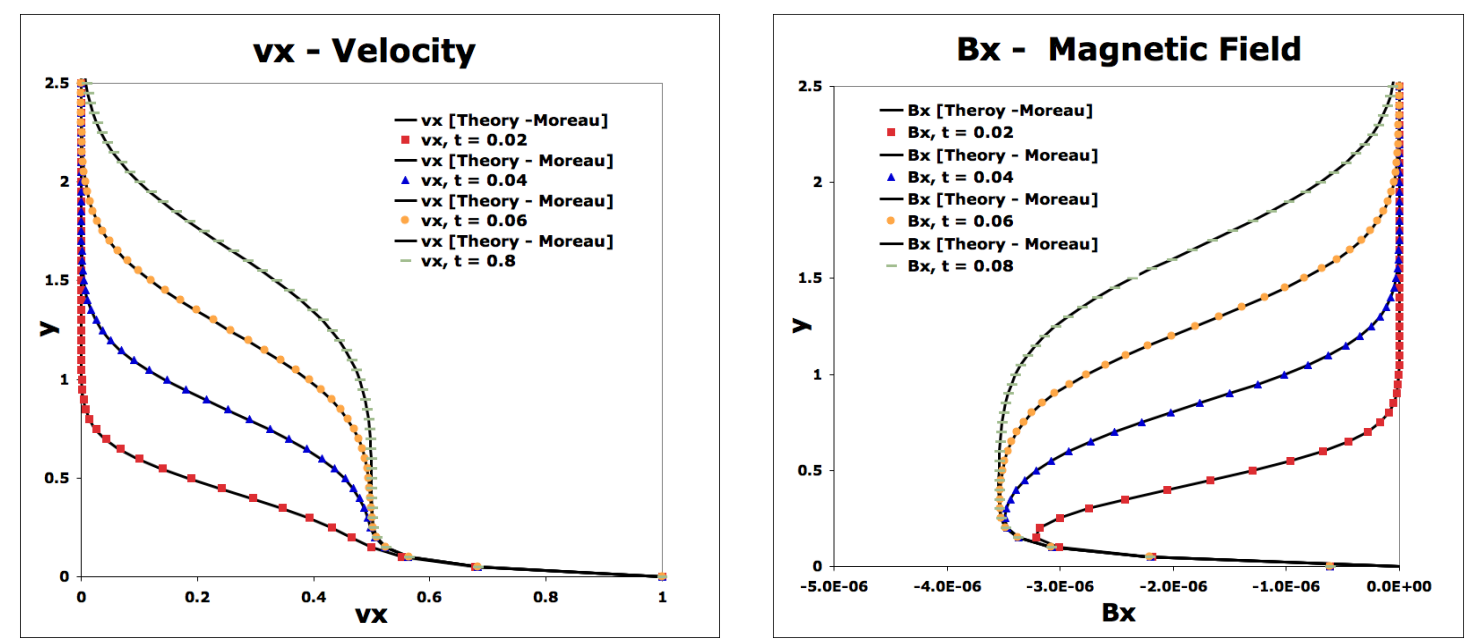

Fig. 8. Modified Rayleigh flow and Alfven wave verification problem. Figures show plots of $x$-velocity (left) and $x$-magnetic field (right). The mesh has $50 \times 250$ elements and the time step is $5 \times 10^{-4}$ time units.

\subsection{Prototype Resistive MHD Problems and Linear Solver Performance}

This section briefly describes three prototype resistive MHD problems that represent a more challenging test of the stabilized FE 2D vector potential formulation and the fully-implicit, fully-coupled solution methods presented in this paper. The first problem is a steady-state MHD duct flow type problem that represents an idealized Faraday conduction pump. The MHD pump induces flow by the action 


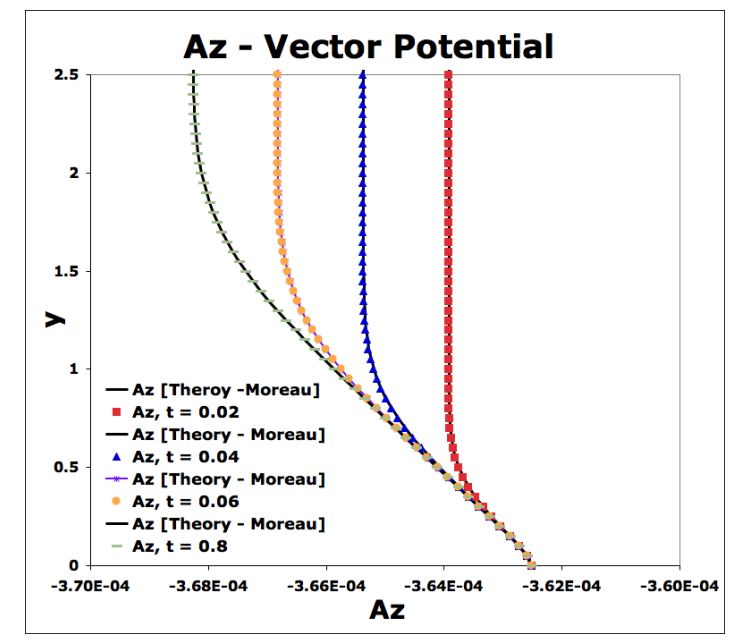

Fig. 9. Modified Rayleigh flow and Alfven wave verification problem. Figure shows plots of $z$-vector potential. The mesh has $50 \times 250$ elements and the time step is $5 \times 10^{-4}$ time units.
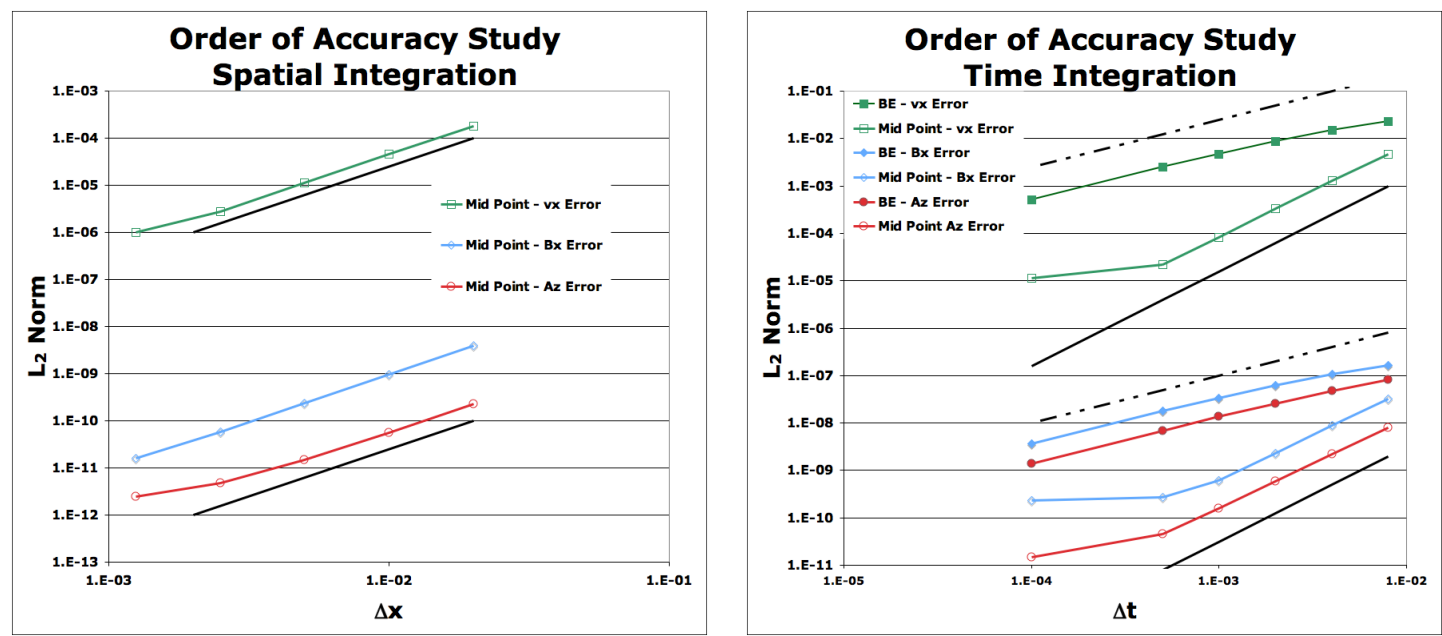

Fig. 10. Order of accuracy study for modified Rayleigh flow and Alfven wave verification problem. A spatial convergence study for $A_{z}, v_{x}, B_{x}$, is shown on the left with a comparison to second order reference lines for $\Delta t=1.0 \times 10^{-4}$. The expected order-of-accuracy is obtained followed by a region where the temporal integration error begins to dominate. Figure on the right shows convergence of the computed numerical solution for the Backward Euler (BE) and midpoint rule (Mid Point) time integrator for $\Delta x=5.0 \times 10^{-3}$. In the Mid Point rule results, the error in the spatial discretization begins to dominate at small time step sizes.

of an applied magnetic and electric field. The second is a coupled thermal-convection buoyancy-induced flow that is modulated by an externally applied magnetic field and produces internally generated fields. The corresponding linear stability problem is the classical hyrdromagnetic Rayleigh-Bernard problem. The final example is a driven magnetic reconnection problem where a Fadeev magnetic field equilibrium [113], which features islands embedded in a Harris current sheet, that undergoes a transient reconfiguration of the magnetic field. The computational timing results presented in this section were obtained on the Red Storm Cray XT3/4 computer at Sandia National Laboratories.

\subsubsection{An Idealized MHD Faraday Pump}

As an illustration of the parallel performance of the one-level additive Schwarz domain decomposition and multilevel preconditioners, a weak scalability study is presented for a 2D idealized Faraday MHD conduction pump. This example problem models an MHD pump that induces flow in a conducting fluid 
by applying an external magnetic field in the $y$-direction and an electric field in the $z$-direction. The domain is $\Omega=[0,10] \times[-1,1]$. There are no-slip fluid velocity conditions applied on the upper and lower surfaces with natural boundary conditions for the system applied at both the inlet and outlet of the domain. On the lower and upper surfaces a constant external magnetic field $\mathbf{B}=\left(0, B_{0}, 0\right)$ is applied by application of a linear variation in $A_{z}$ in the $x$-direction in the range of $x \in[2.5,7.5]$ while outside of this range the magnetic field is zero. A constant electric field, $E_{z}^{0}$ is applied in the $z$-direction. The interaction of these fields produces a Lorentz force that pulls fluid in from the $x=0$ boundary with a parabolic profile, contorts the velocity field into a common " $\mathrm{M}$ " profile for these types of flows [114-116], and then the flow exits with a parabolic profile (see Figure 11 and 12). The simple geometry of this problem facilitates scalability studies as different mesh sizes can be easily generated.

Additive Schwarz domain decomposition (DD) methods [117,118,33] partition the original domain into subdomains and approximately solve the discrete problems corresponding to the individual subdomains in parallel. Among Schwarz schemes, there are a number of choices which can greatly affect the overall solution time and robustness. These choices include the subdomain size, the amount of overlap between subdomains, and the partitioning metric which can alter the shape and aspect ratio of subdomains (see e.g.[119,33,120,121,70]). The choices also include the selection of subdomain solver such as an incomplete LU factorization (ILU) (with further options for dropping nonzeros in the factorizations and ordering equations within a subdomain [122]), and the introduction of a coarse grid solve [123,124]. In our numerical study, we briefly consider the effect of ILU fill-in for the one-level preconditioner and the benefit of employing multilevel methods with coarse operator solves as described above.

As an initial illustration of parallel efficiency we consider the weak scaling of the one-level DD ILU preconditioner as presented in Table 4 . In this study the 16 processor case solves the problem on a $64 \times 64$ mesh. The weak scaling study keeps the work per processor fixed as the problem size is increased. This study is for a low $R e=R e_{m}=0.7$ flow with $H a=1$. The Krylov method is a non-restarted GMRES technique to allow only the parallel scalability of the preconditioners to be addressed. For the one-level DD preconditioner, an incomplete factorization ILU $(\mathrm{k})$ sub-domain solver was used with $k=1,3,7$. For the 3-level preconditioner, the fine and medium meshes use an ILU(1) smoother and the coarsest problem was solved by the KLU sparse direct solver.
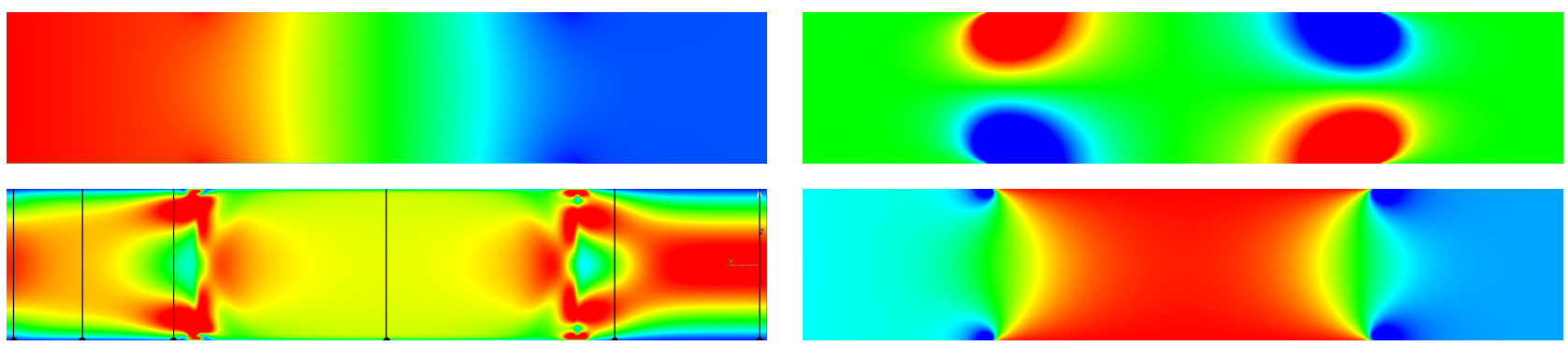

Fig. 11. Contour plots of $A_{z}$ (upper left), velocity vector magnitude (lower left), $B_{x}$ (upper right) and $B_{y}$ (lower right) for idealized Faraday conduction MHD pump.

Figure 13 graphically presents the parallel and algorithmic scaling of the one- and three-level preconditioners for the MHD Faraday pump presented in Table 4. Figure 13 (left) summarizes the results for the average iteration count per Newton step as a function of problem size. As the number of unknowns, $N$ (as well as the number of processors, $P$, in this scaled study), is increased, the number of iterations to convergence for the one-level schemes increases significantly: roughly $N^{1 / 2}$ in two dimensions. Note that an optimal convergence property, that is an iteration count independent of problem size, is roughly obtained for the 3-level preconditioner. On the coarsest level, a serial sparse matrix direct solver, KLU, was used to factor the coarse matrix. Since the fine grid smoother is highly parallel and the fine grid 


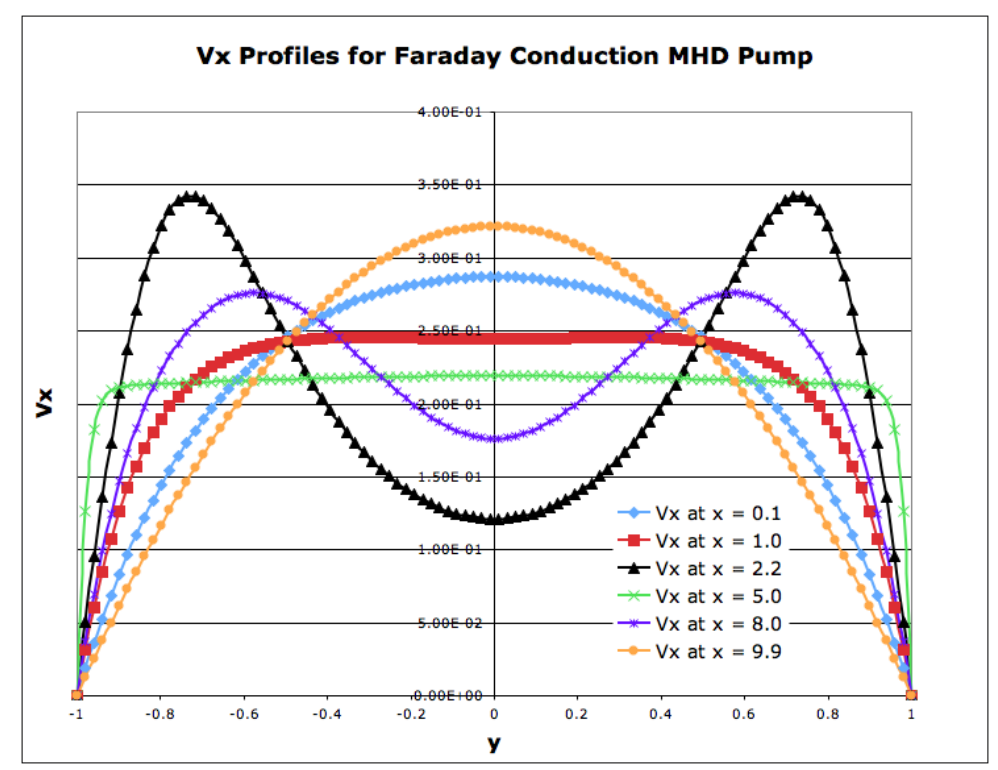

Fig. 12. $V_{x}$ velocity profiles for the developing fow in an idealized Faraday MHD pump. The locations of the profiles are shown graphically in Fig. 11.

\begin{tabular}{|c|c|c|c|c|c|c|c|c|c|}
\hline \multirow[t]{2}{*}{ proc } & \multirow{2}{*}{$\begin{array}{l}\text { fine } \\
\text { unks }\end{array}$} & \multicolumn{2}{|c|}{ 1-level ILU(1) } & \multicolumn{2}{|c|}{ 1-level ILU(3) } & \multicolumn{2}{|c|}{ 1-level ILU(7) } & \multicolumn{2}{|c|}{ 3-level V $(1,1)$} \\
\hline & & $\begin{array}{r}\text { avg its/ } \\
\text { Newt } \\
\text { step }\end{array}$ & $\begin{array}{l}\text { time } \\
\text { (sec) }\end{array}$ & $\begin{array}{r}\text { avg its/ } \\
\text { Newt } \\
\text { step }\end{array}$ & $\begin{array}{l}\text { time } \\
(\mathrm{sec})\end{array}$ & $\begin{array}{r}\text { avg its/ } \\
\text { Newt } \\
\text { step }\end{array}$ & $\begin{array}{l}\text { time } \\
\text { (sec) }\end{array}$ & $\begin{array}{r}\text { avg its/ } \\
\text { Newt } \\
\text { step }\end{array}$ & $\begin{array}{l}\text { time } \\
\text { (sec) }\end{array}$ \\
\hline 16 & $256 \mathrm{~K}$ & $136[9]$ & 9.8 & $86[8]$ & 8.6 & $62[9]$ & 9.7 & $60[8]$ & 7.5 \\
\hline 64 & $1 \mathrm{M}$ & $313[9]$ & 22.8 & $198[9]$ & 15.1 & $154[9]$ & 14.8 & $106[9]$ & 9.8 \\
\hline 256 & $4 \mathrm{M}$ & $714[11]$ & 82.4 & $701.4[11]$ & 44.9 & $392[8]$ & 36.3 & $189[9]$ & 15.6 \\
\hline 1024 & $16 \mathrm{M}$ & $1583[10]$ & 318 & $1158[12]$ & 191 & $888[10]$ & 123 & $174[10]$ & 16.6 \\
\hline 4096 & $64 \mathrm{M}$ & $2667[20]$ & 750 & $1951[16]$ & 488 & $1766[14]$ & 429 & $178[13]$ & 34.8 \\
\hline
\end{tabular}

Table 4

Comparison of 1-level and 3-level scheme for MHD Faraday pump example problem. The 3-level method uses an aggregation size of 80 and an ILU(1) smoother on the fine and medium meshes with the KLU direct solver on the coarsest problem. The table entry above for 136[9] indicates the number of GMRES iterations followed by the number of Newton steps in brackets.

work per processor is roughly constant, the cost of producing the coarse grid problem, and executing the direct solve (KLU) on the increasingly larger coarse grid, causes an increase in the CPU time for the larger problems. While this loss of CPU time scaling is non-optimal, it must be pointed out that the 3-level method is still significantly faster (a factor of about 10-20x) than the corresponding one-level methods. To mitigate this growth of CPU time for the coarse grid solve either approximate coarse grid methods can be used (e.g. [70]) or more levels could be employed.

As a proof-of-capability of the multilevel preconditioner, an initial demonstration of the solution of a very large scale problem is presented that is solved on tens-of-thousands of processors. Specifically a problem with $1+$ billion unknowns for the MHD pump is solved on the Cray XT3/4 using 6,000 nodes with 4 cores per node for a total of 24,000 cores. For this problem size the one-level method cannot be applied. The details of this calculation are presented in Table 5. These results are very encouraging. 

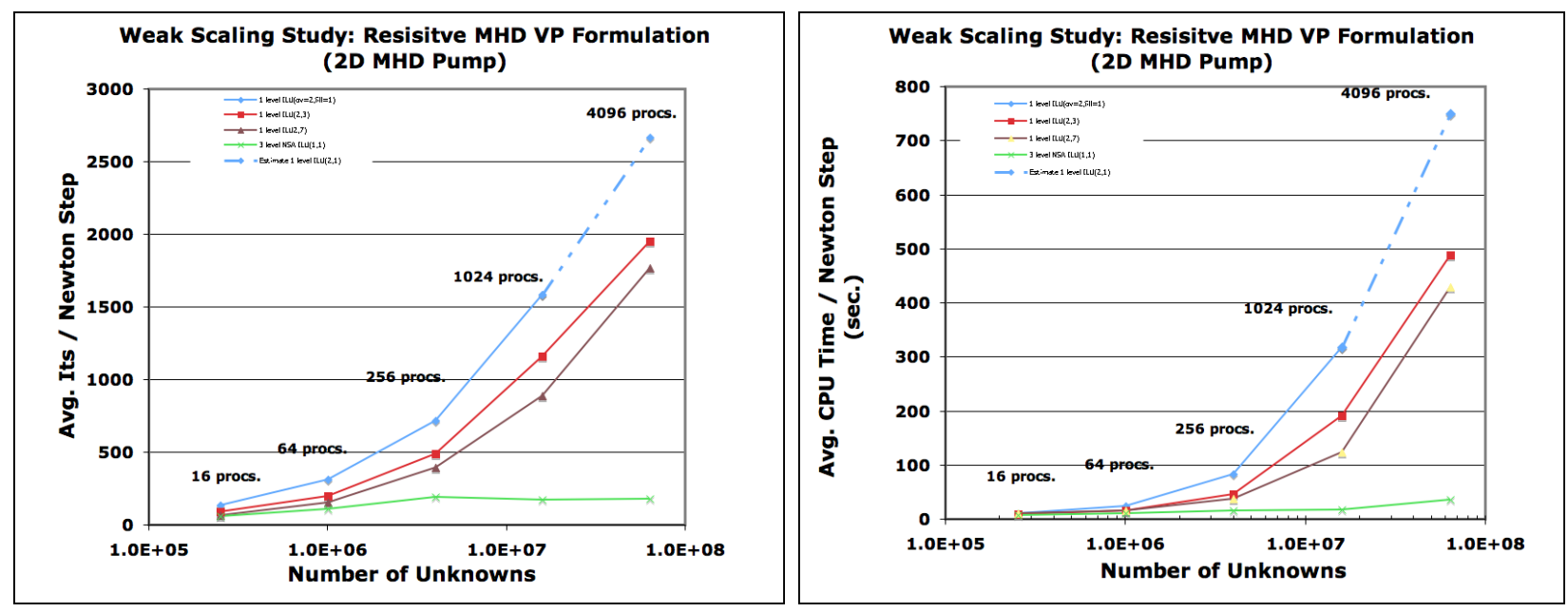

Fig. 13. Weak scaling results for MHD Faraday pump problem. The scaling of the average number of iterations per Newton step (left) and the average CPU time per Newton step (right).

They indicate convergence of the Newton iteration in $O(10)$ iterations and of the linear solver in $O(100)$, values that are not too far from the more complete weak scaling study of Table 4 that employes three levels and a larger aggregation size of 80 . Finally the ability of the multilevel preconditioned NewtonKrylov solver to scale to $1+$ billion unknowns on 24,000 cores is an indication that the underlying solution strategy is reasonably robust and scalable for this low $R e_{m}$ prototype problem.

Table 5

\begin{tabular}{|c|c|c|c|c|c|c|c|}
\hline cores & $\begin{array}{c}\text { Fine Mesh } \\
\text { Level 0 } \\
\text { Unkns. }\end{array}$ & $\begin{array}{c}\text { Intermed. } \\
\text { Level 1 } \\
\text { Unkns. }\end{array}$ & $\begin{array}{c}\text { Intermed. } \\
\text { Level 2 } \\
\text { Unkns. }\end{array}$ & $\begin{array}{c}\text { Coarse } \\
\text { Level 3 } \\
\text { Unkns. }\end{array}$ & $\begin{array}{c}\text { Newton } \\
\text { Its. }\end{array}$ & $\begin{array}{c}\text { Avg. No. } \\
\text { Linear Its. / } \\
\text { Newton }\end{array}$ & $\begin{array}{c}\text { Total } \\
\text { Time } \\
\text { (min.) }\end{array}$ \\
\hline \hline 24,000 & $1+$ Billion & $23.3 \mathrm{M}$ & $.5 \mathrm{M}$ & $11.2 \mathrm{~K}$ & 18 & 86 & 33 \\
\hline
\end{tabular}

Details of $1+$ billion unknown MHD Faraday pump calculation. The simulation used a mesh of size $51,200 \times$ 5,120 with roughly $260 \mathrm{M}$ quad elements. The multilevel preconditioner used an aggregation size of 45 at each level to produce the coarsened operators.

\subsubsection{A Classical Hydromagnetic Rayleigh-Bernard Stability Computation}

This challenging resistive MHD prototype problem consists of a buoyancy induced thermal convection flow that is modulated by the existence of an externally applied magnetic field. It combines the classical Rayleigh-Bernard buoyancy induced thermal convection flow problem with an externally applied B field. The magnetic field induces Maxwell stresses that add additional stabilizing effects to the traditional damping provided by the viscous forces. These coupled mechanisms are, for example, critical components of large-scale geo-dynamo simulations that model the time dependent behavior of the Earth's magnetic field (e.g.[125,1]). This problem solves for the unknowns $\left(\mathbf{v}, P, T, A_{z}\right)$ from the system outlined in Table 1 where the density variation is modeled by a Boussinesq approximation [125]. The rectangular domain for this problem is $\Omega=[0,10] \times[0,1]$. There are no-slip fluid velocity conditions applied on the upper and lower surfaces with natural boundary conditions for the system applied at both the left and right boundary of the domain. A temperature difference is maintained in the vertical direction by holding the lower surface to a high temperature $T_{H}$ and the upper surface at the lower temperature $T_{C}$ that is separated by a distance $d$. This temperature difference $\left(\Delta T=\left(T_{H}-T_{C}\right)\right)$ produces an unstable density stratification that interacts with gravity in the negative $y$-direction. In the classical 
Rayleigh-Bernard problem, flow is induced when the non-dimensional $R a=\rho^{2} C_{p} g \beta \Delta T d^{3} /(\mu \lambda)$ becomes sufficiently large to have buoyancy effects overwhelm the stabilizing viscous forces [125]. In the case of the hydromagnetic Rayleigh-Bernard (HMRB) problem a stabilizing constant external magnetic field $\mathbf{B}=\left(0, B_{0}, 0\right)$ is applied on the lower and upper surfaces. For a thermal convecting flow at fixed Rayleigh number, convective flow will be damped when the non-dimensional magnetic field strength is increased beyond a critical Chandrasekhar number, $Q=H a^{2}$. Beyond this limit, the Maxwell stresses existing in the curved magnetic field lines shut down the convective cellular flow. A typical stable nonlinear solution where cellular flow and fields exist is presented in Figure 14.

As an example of the robustness and efficiency of the fully-coupled multi-level preconditioned NK solution technology, we present the results in Figure 15 for convergence of the nonlinear Newton iteration (left image) and the preconditioned Krylov linear solver (right image). The convergence of the outer fully-coupled nonlinear Newton solver in Figure 15 (left) indicates that, as the Chandrasekhar number increases, the nonlinearity of the problem increases and the convergence of the nonlinear iteration becomes more difficult. Without the use of backtracking techniques [75], convergence is not obtained past $Q=4$. Beyond $Q=11$, a direct-to-steady-state solution was not obtained and the use of continuation and/or transient solution methods would need to be considered. In the context of the linear solver convergence, Figure 15 (right) indicates that the multilevel preconditioned Krylov method converges substantially faster to the solution in the first two Newton steps. In addition, the ability of the 3-level methods to provide a sufficient linear residual decrease in the sub-problems for Newton's method allows for a more robust iterative nonlinear solver. This is indicated in Table 6 for the hydromagnetic Rayleigh-Bernard problem. Note that the 1 level DD preconditioner failed to solve this problem in less than 40 Newton steps since the linear solver failed to reach the linear solver convergence criteria of $\eta=10^{-3}$ in 2000 iterations, for 36 out of 40 Newton steps. We have seen many examples for which a direct-to-steady-state nonlinear calculation will fail, for very ill-conditioned large linear systems, because the iterative linear solver converges too slowly to be practical or does not converge at all.
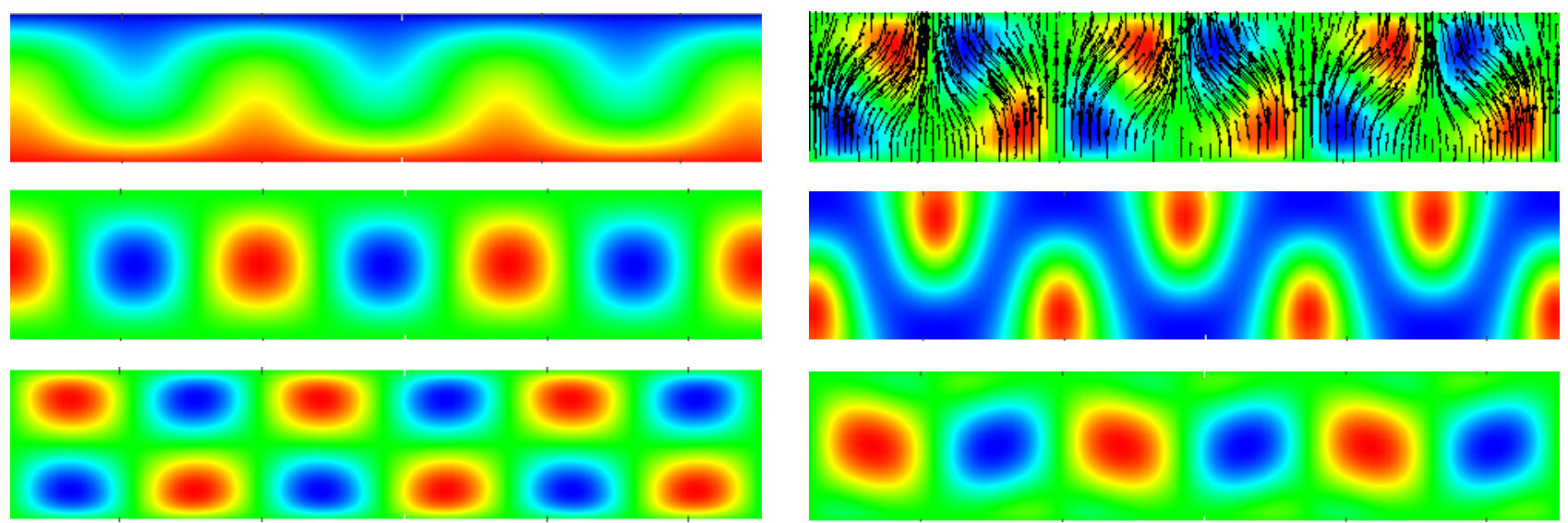

Fig. 14. Contour plots for the hydromagnetic Rayleigh-Bernard stability type calculation. The images show the stable nonlinear flow and fields at $R a=2500$ and $Q=9$ for temperature (upper left), $V_{y}$ (center left), $V_{x}$ (lower left), and $J_{z}$ with the magnetic field vectors (upper right), $B_{y}$ (center right), and $B_{x}$ (lower right).

As an additional verification of the accuracy of the resistive MHD formulation presented above, we present a comparison of the computed critical Rayleigh number from simulation, and the theoretical value in Chandrasekhar [125]. In this study a computational eigenvalue analysis of the linear stability problem is computed as in [84] with the results summarized in Table 7, where the accuracy of the formulation is clearly apparent. Finally, by employing the direct-to-steady-state solution capability 
based on Newton-Krylov type techniques as presented in this study, additional advanced solution methods such as parameter continuation, bifurcation tracking and automated linear stability analysis algorihtms can be effectively developed to analyze the complex nonlinear solution spaces for the HMRB problem.
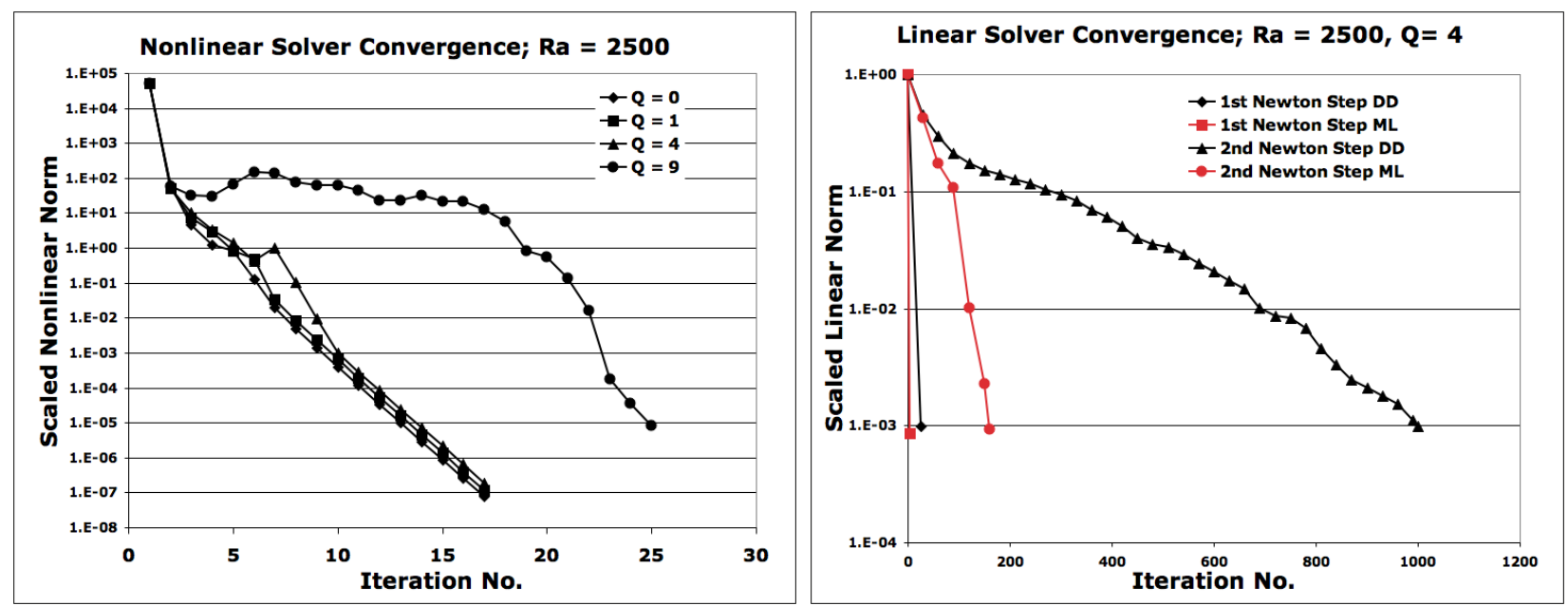

Fig. 15. Hydromagnetic Rayleigh-Bernard problem. A convergence plot of the nonlinear scaled norm in Newton's method with the ML preconditioner (left) and the linear scaled norm in non-restarted GMRES for DD and ML preconditioner (right).

Table 6

\begin{tabular}{|c|c|c|r|r|r|r|r|r|}
\hline \multirow{2}{*}{ proc } & fine grid & fine grid & \multicolumn{2}{|c|}{ 1-level ILU } & \multicolumn{3}{|c|}{ 3-level V(1,1) ILU-ILU-KLU } \\
\cline { 4 - 9 } & size & unknowns & avg its/ & total & medium & coarse & avg its/ & total \\
& & & Newt & time & unkns & unkns & Newt & time \\
& & & step & $(\mathrm{sec})$ & size & size & step & $($ sec $)$ \\
\hline \hline 2048 & $500 \times 5000$ & $12.5 \mathrm{M}$ & $1910[40]$ & $>7200^{*}$ & 412450 & 13745 & $115[17]$ & 226 \\
\hline
\end{tabular}

Comparison of convergence for the 1-level and 3-level preconditioner for Hydromagnetic Rayleigh-Bernard problem. ${ }^{*}$ The 1 level DD preconditioner failed to solve this problem in less than 40 Newton steps.

Table 7

\begin{tabular}{|r|r|c|c|}
\hline$Q$ & $R a^{*}$ & $R a_{c r}[$ Chandrasekhar $[125]]$ & $\%$ error \\
\hline 0 & 1707.77 & 1707.8 & 0.002 \\
\hline $10^{1}$ & 1945.78 & 1945.9 & 0.006 \\
\hline $10^{2}$ & 3756.68 & 3757.4 & 0.02 \\
\hline
\end{tabular}

Comparison of the computed critical Rayleigh number, $R a^{*}$, by a computational linear stability eigenvalue analysis with theory, $R a_{c r}$, from Chandrasekhar [125]. The mesh is a rectangular $200 \times 2000$ mesh.

\subsubsection{A Transient Simulation of Driven Magnetic Reconnection: Island Coalescence}

As an example of a current scientifically relevant, and computationally challenging simulation, the numerical and computational performance of the implicit stabilized FE resistive MHD is applied to an example of driven magnetic reconnection. Magnetic reconnection is a fundamental process whereby 
a sheared magnetic field topology is altered via some dissipation mechanism, resulting in a rapid conversion of magnetic field energy into plasma energy and significant plasma transport. Magnetic reconnection dominates the energetics and dynamics of many space and laboratory plasmas, and is at the root of explosive phenomena such as solar flares, coronal mass ejections, plasmoid ejection from the earth's magnetotail, and major disruptions in magnetic fusion energy (MFE) experiments [126]. However, plasmas in all the above-mentioned cases are known to have negligible electrical resistivity. This negligible resistivity cannot explain the reconnection timescales observed in nature, and this issue has drawn significant attention over the last 50 years [126]. Furthermore, this small magnitude of resistivity forces reconnection to occur in space in very thin current sheets and in rapid stability changing events that are very difficult to resolve. Recently the development of an efficient implicit resistive MHD capability in a mapped structured mesh finite volume context has led to new computational scientific results that have elucidated the exisitence of an asymptotic low resistivity reconnection rate regime that follows the theoretical Sweet-Parker $[126,127]$ scaling. The key to the computational verification of this regime is the ability to effectively time-integrate the multiple-time-scale resistive MHD system [127].

As a final illustration of the accuracy and performance of the fully-implicit resistive MHD formulation described above, a set of computations that were previously carried out with the fully-implicit mapped FV capability in [127] are presented. The transient island coalescence problem consists of a perturbed Harris sheet magnetic field configuration [126,127] that introduces two magnetic islands in the plasma as initial conditions for the island coalescence problem. The structure of this perturbation can be seen in the initial condition plot at time $t=0$ of Figure 16 (upper left) with iso-lines of $A_{z}$. The combined magnetic field produced by the two magnetic islands produces Lorentz forces that pulls the islands together, and at finite resistivity the islands coalesce (join) to form one island. Figure 16 shows three iso-line plots of $A_{z}$ and filled color contours of the the plasma current $J_{z}$ during the reconnection event. Clearly evident is the formation of the x-point between the islands (see upper right image), the development of a thin current sheet at that same x-point location, and the movement of the center of the islands (o-points) towards the x-point [126,127]. The dynamics of island coalescence changes as a function of resistivity. For larger resistivities the $\mathrm{x}$ - and o-points monotonically approach each other, for low resistivities fluid-plasma pressure builds up as the islands approach and a sloshing or bouncing of the o-point position is encountered that leads to lower reconnection rates (for more details on the physics see e.g. [126,128]). Next a very brief description of the island coalescence problem is presented with details provided in [127].
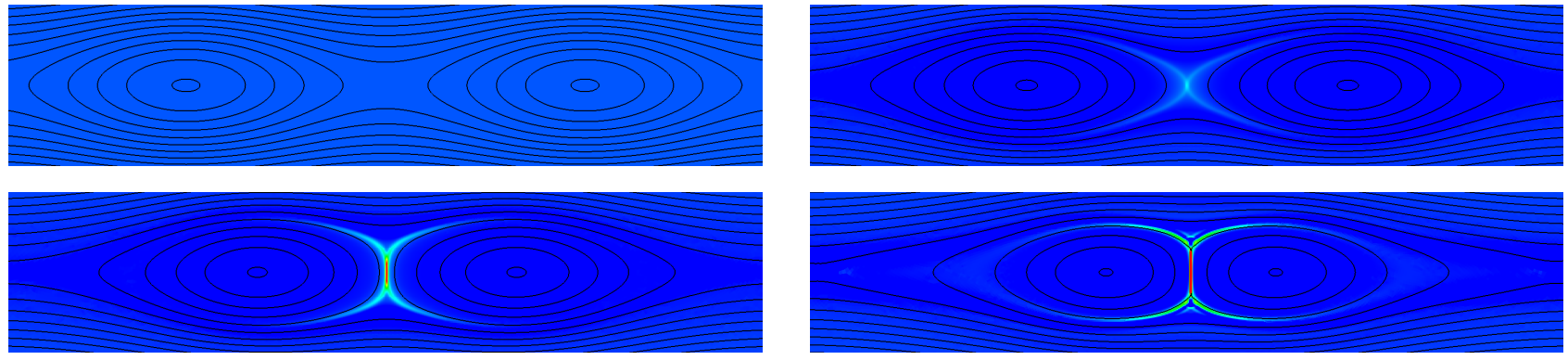

Fig. 16. Contour plots for the island coalescence driven magentic reconnection at times $t=0.0,9.0,10.0,12.6$ computed on the unstructured mesh above. The images show isolines of the magnetic potential $A_{z}$ and filled contours of the current $J_{z}$.

The initial conditions for the island coalescence problem consists of zero fluid velocities $\left(\mathbf{v}^{0}=0\right)$, and a Fadeev magnetic equilibrium [113]. The description of this problem is in the context of the magnetic 
vector potential. The initial conditions, $A_{z}^{0}$, and the resulting balancing plasma fluid pressure, $P^{0}$ are given by

$$
\begin{gathered}
A_{z}^{0}(x, y, 0)=\delta \ln \left[\cosh \left(\frac{y}{\delta}\right)+\epsilon \cos \left(\frac{x}{\delta}\right)\right], \\
P^{0}(x, y, 0)=P_{0}+\frac{\left[1-\epsilon^{2}\right]}{2\left[\cosh \left(\frac{y}{\delta}\right)+\epsilon \cos \left(\frac{x}{\delta}\right)\right]^{2}},
\end{gathered}
$$

where $\delta=1 /(2 \pi)$ and $P_{0}=1.0$. To assure that the initial condition is a resistive equilibrium, an external applied electric field, $E_{z}^{0}$ of the form

$$
E_{z}^{0}(x, y)=\frac{\eta\left[1-\epsilon^{2}\right]}{\delta\left[\cosh \left(\frac{y}{\delta}\right)+\epsilon \cos \left(\frac{x}{\delta}\right)\right]^{2}}
$$

in the $z$-direction is included. The boundary conditions on velocity consist of zero normal velocities on each surface and zero tangential stress. On the upper boundary, the vector potential $A_{z}$ is set by Equation (24), and $P_{0}=1$.

Our interest in this brief study is to compute the peak magnetic reconnection rate as a function of the fluid resistivity as in [127] for a range of values that includes the fast and slow (Sweet-Parker) reconnection regimes. The peak magnetic reconnection rate in this simulation is given by the maximum value of the time derivative of the vector potential at the x-point, i.e. $\Psi_{t} \equiv \partial A_{z} / \partial t$ for all time. Figure 17 (left) presents the result of a set of stabilized FE simulations to determine the reconnection rate at the $\mathrm{x}$-point for 3 different resistivity values that span the fast and slow reconnection regimes. Clearly evident in this figure is the peak value for each time history and the oscillatory (or sloshing) behavior of the reconnection rate for lower resistivities as in [127]. In Figure 17 (right) the scaling of the peak magnetic reconnection rate as a function of the resistivity is presented. The magnetic Reynolds number varies from 50 to $10^{5}$. The FE results are seen to compare very well with the results of Knoll and Chacon [127]. In this plot, results are presented for non-uniform structured meshes of size 130,000 FE nodes with a distribution of $512 \times 256$ in $(x, y)$. In this mesh the FE elements are clustered by the $\mathrm{x}$-point and geometrically increase in size away from this point, this roughly corresponds to the meshes used in [127]. In addition, results for an unstructured mesh with 40,000 FE nodes, that highly resolves the $\mathrm{x}$-point region with a uniform mesh, and then transitions to a coarse unstructured mesh away for the $\mathrm{x}$-point are presented as well. A graphic of an unstructured mesh and the iso-lines of $A_{z}$ is presented in Figure 18. The stabilized FE results are demonstrated to closely correspond to the mapped FV results for both the structured and unstructured meshes. In addition, the existence of the theoretical Sweet-Parker slow magnetic reconnection regime were $\Psi_{t} \approx \sqrt{\eta}$ is also confirmed. A power law fit to the FE results indicate a 0.48 power scaling over this interval.

A preliminary study of the scaling for the multilevel preconditioner for the transient solution of the island coalescence problem at $\eta=10^{-3}$ is presented in Table 8. Here, factoring out the increase in nonlinear iterations per time step, the general block aggregation based algebraic multilevel preconditioner appears to be reasonably effective at keeping the growth in iterations per time step under control as the mesh size is increased. While the scaling of the multilevel preconditioned Newton-Krylov method is not optimal with problem size, the increase in the number of linear iterations per Newton step is gradual with the problem size, and represents a reasonable step towards a scalable algebraic multilevel method. 

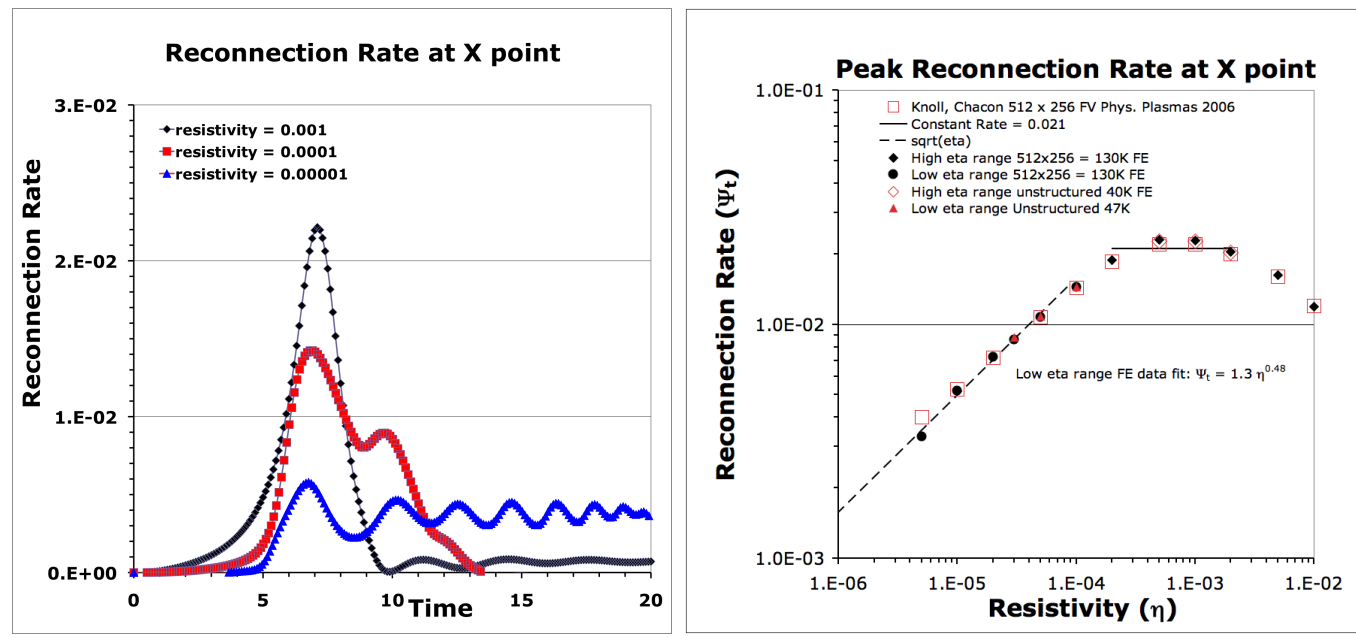

Fig. 17. Left: Time history of the reconnection rate at the $\mathrm{x}$-point in the island coalescence problem for various resistivities. Oscillations in the reconnection rate are evident for lower resistivities as a consequence of the sloshing of magnetic islands. Right: Peak resistive magnetic reconnection rate for the island coalescence problem. Graph includes data for stabilized FE formulation and a comparison to FV resistive MHD data from Knoll and Chacon [127].

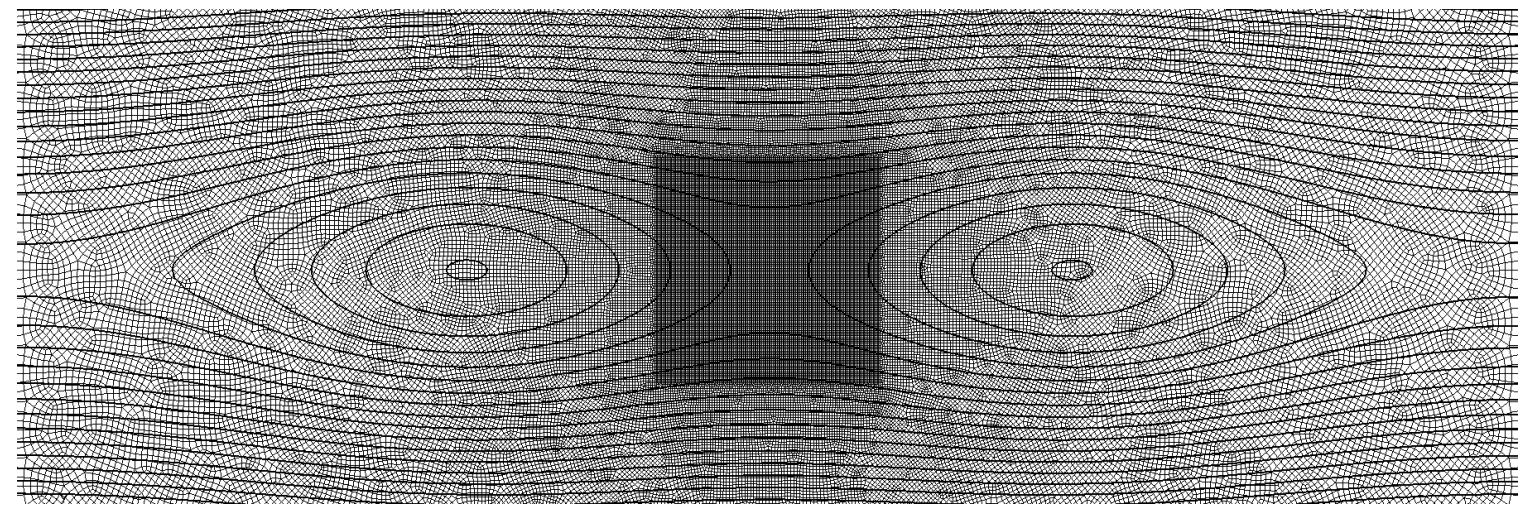

Fig. 18. Detail of unstructured mesh for island coalescence problem. This image also has iso-lines of $A_{z}$ represented at time $t=9.0$.

Table 8

\begin{tabular}{|r|c|c|r|r|c|c|c|}
\hline Procs & Mesh & $N_{\text {unks }}$ & $\begin{array}{r}\text { Newton/ } \\
\Delta t\end{array}$ & $\begin{array}{c}\text { Gmres/ } \\
\text { Newton }\end{array}$ & $\begin{array}{c}\text { Time/ } \\
\text { Newton }\end{array}$ & $\begin{array}{c}\text { Gmres/ } \\
\Delta t\end{array}$ & $\begin{array}{c}\text { Time/ } \\
\Delta t\end{array}$ \\
\hline \hline 1 & $64 \times 64$ & $16 \mathrm{~K}$ & 3.9 & 4.4 & 2.1 & 17.2 & 8.1 \\
\hline 4 & $128 \times 128$ & $64 \mathrm{~K}$ & 4.6 & 5.8 & 2.6 & 26.7 & 11.9 \\
\hline 16 & $256 \times 256$ & $0.25 \mathrm{M}$ & 4.9 & 6.3 & 2.9 & 30.9 & 14.2 \\
\hline 64 & $512 \times 512$ & $1 \mathrm{M}$ & 6.2 & 8.8 & 4.0 & 54.6 & 24.6 \\
\hline
\end{tabular}

Weak scaling of the block AMG preconditioned Newton-Krylov solver for the stabilized resistive MHD formulation on the island coalescence problem. The time step is $\Delta t=0.1$ time units. The smoother in the Petrov-Galerkin smoothed aggregation method is an ILU(1) and it is a V(1,1) cycle.

\section{Conclusions}

This paper has presented an initial study of an unstructured fully-implicit stabilized FE formulation for low-Mach-number resistive MHD. This solution methods used in this formulation are based 
on a fully-coupled Newton-Krylov solver that employs algebraic multilevel prconditioners. This initial study included verification and order-of-acuracy results that verified the expected convergence rates for the 2D vector potential form of this system. As an illustration of the robustness, scalability and efficiency of the solution techniques representative results were presented for a MHD duct flow, a hydromagnetic Ralyeigh-Bernard linear stability calculation and a challenging magnetic island coalescence problem. The results demonstrated the robustness of the underlying fully-coupled Newton-Krylov nonlinear solver on the steady-state MHD pump and the hydro-magnetic Rayleigh Bernard linear stability calculation as well as the fully-implicit solution of the island coalescence problem. The efficiency and scalability results of this study were very encouraging and demonstrated the increased convergence rate of the block aggressive coarsening, fully-coupled parallel multilevel preconditioner over more standard parallel additive Schwarz domain decomposition methods. In future work, the fully-coupled fully-implicit solution methods in this study will be developed and evaluated for a full 3D resistive MHD solver.

\section{Acknowledgments}

The authors wish to thank Gary Hennigan and Rob Hoekstra for their collaborative effort in developing the simulation code framework that is used as the software development platform. We also thank Dana Knoll for helpful discussions on the MHD equations and magnetic reconnection phenomena, Pavel Bochev for discussions on finite element representations for MHD and John Evans for helpful discussions on stabilization techniques.

\section{References}

[1] H. Goedbloed, S. Poedts, Principles of Magnetohydrodynamics with Applications to Laboratory and Astrophysical Plasmas, Cambridge Univ. Press, 2004.

[2] K. E. Brenan, S. L. Campbell, L. R. Petzold, Numerical Solution of Initial-Value Problems in DifferentialAlgebraic Equations, SIAM, Classics in Applied Math, 1996.

[3] U. M. Ascher, L. R. Petzold, Computer Methods for Ordinary Differential Equations and DifferentialAlgebraic Equations, SIAM, 1998.

[4] W. Dai, P. R. Woodward, On the divergence-free condition and conservation laws in numerical simulations for supersonic magnetohydrodynamic flows, Astrophys. J. 494 (1998) 317.

[5] W. Dai, P. R. Woodward, A simple finite difference scheme for multidimensional magnetohydrodynamic equations, J. Comput. Phys. 142 (1998) 331.

[6] D. Ryu, F. Miniati, T. W. Jones, A. Frank, A divergence-free upwind code for multi-dimensional magnetohydrodynamics flows, Astrophys. J. 509 (1998) 244.

[7] D. S. Balsara, D. S. Spicer, A staggered mesh algorithm using high order Godunov fluxes to ensure solenoidal magnetic fields in magnetohydrodynamics simulations, J. Comput. Phys. 149 (1999) 270-292.

[8] D. S. Balsara, Divergence-free adaptive mesh refinement for magnetohydrodynamics, J. Comput. Phys. 174 (2001) 614-648.

[9] G. Tóth, The $\nabla \cdot \mathbf{B}=\mathbf{0}$ constraint in shock-capturing magnetohydrodynamics codes, J. Comput. Phys. 161 (2000) 605-652. 
[10] G. Tóth, R. Keppens, M. A. Botchev, Implicit and semi-implicit schemes in the Versatile Advection Code: numerical tests, Astron. Astrophys. 332 (1998) 1159-1170.

[11] R. Keppens, G. Tóth, M. A. Botchev, A. V. D. Ploeg, Implicit and semi-implicit schemes: algorithms, Int. J. Numer. Meth. Fluids 30 (1999) 335-352.

[12] A. Y. Aydemir, D. C. Barnes, An implicit algorithm for compressible three-dimensional magnetohydrodynamic calculations., J. Comput. Phys. 59 (1) (1985) 108 - 19.

[13] W. Park, J. Breslau, J. Chen, G. Y. Fu, S. C. Jardin, S. Klasky, J. Menard, A. Pletzer, B. C. Stratton, D. Stutman, H. R. Strauss, L. E. Sugiyama, Nonlinear simulation studies of tokamaks and sts., Nuclear fusion 43 (6) (2003) $483-9$.

[14] S. C. Jardin, J. A. Breslau, Implicit solution of the four-field extended-magnetohydrodynamic equations using high-order high-continuity finite elements., Phys. Plasmas 12 (5) (2005) 056101.

[15] D. S. Harned, W. Kerner, Semi-implicit method for three-dimensional compressible magnetohydrodynamic simulation, J. Comput. Phys. 60 (1985) 62-75.

[16] D. S. Harned, D. D. Schnack, Semi-implicit method for long time scale magnetohydrodynamic computations in three dimensions, J. Comput. Phys. 65 (1986) 57-70.

[17] D. D. Schnack, D. C. Barnes, D. S. Harned, E. J. Caramana, Semi-implicit magnetohydrodynamic calculations, J. Comput. Phys. 70 (1987) 330-354.

[18] D. S. Harned, Z. Mikic, Accurate semi-implicit treatment of the Hall effect in magnetohydrodynamic computations, J. Comput. Phys. 83 (1989) 1-15.

[19] C. R. Sovinec, A. H. Glasser, T. A. Gianakon, D. C. Barnes, R. A. Nebel, S. E. Kruger, D. D. Schnack, S. J. Plimpton, A. Tarditi, M. S. Chu, the NIMROD team, Nonlinear magnetohydrodynamics simulation using high-order finite elements, J. Comput. Phys. 195 (1) (2004) 355-386.

[20] A. C. Robinson, C. J. Garasi, Three-dimensional z-pinch wire array modeling with alegra-hedp, Computer Physics Communications 164 (2004) 408-413.

[21] A. C. R. et. al., Alegra: An arbitrary lagrangian-eulerian multimaterial, multiphysics code, in: AIAA 2008-1235 46th AIAA Aerospace Sciences Meeting and Exhibit, Reno, NV, 2008.

[22] L. Chacón, D. A. Knoll, J. M. Finn, Implicit, nonlinear reduced resistive MHD nonlinear solver, J. Comput. Phys. 178 (1) (2002) 15-36.

[23] L. Chacón, D. A. Knoll, A 2D high- $\beta$ Hall MHD implicit nonlinear solver, J. Comput. Phys. 188 (2) (2003) 573-592.

[24] A. Hujeirat, IRMHD: an implicit radiative and magnetohydrodynamical solver for self-gravitating systems, Mon. Not. R. Astron. Soc. 298 (1998) 310-320.

[25] A. Hujeirat, R. Rannacher, On the efficiency and robustness of implicit methods in computational astrophysics, New Astronomy Reviews 45 (2001) 425-447.

[26] D. R. Reynolds, R. Samtaney, C. S. Woodward, A fully implicit numerical method for single-fluid resistive magnetohydrodynamics., J. Comput. Phys. 219 (1) (2006) $144-62$.

[27] S. Ovtchinnikov, F. Dobrian, X.-C. Cai, D. Keyes, Additive schwarz-based fully coupled implicit methods for resistive hall magnetohydrodynamic problems, J. Comput. Phys. 225 (2007) 1919 - 1936.

[28] L. Chacón, An optimal, parallel, fully implicit newton-krylov solver for three-dimensional visco-resistive magnetohydrodynamics, Phys. Plasmas 15 (2008) 056103. 
[29] L. Chacón, Scalable solvers for 3d magnetohydrodynamics, J. Physics: Conf. Series 125 (2008) 012041.

[30] D. R. Reynolds, R. Samtaney, C. S. Woodward, Operator-based preconditioning of stiff hyperbolic systems, SIAM J. Sci. Comput.In press.

[31] P. N. Brown, Y. Saad, Convergence theory of nonlinear Newton-Krylov algorithms, SIAM J. Optimization 4 (1994) 297-330.

[32] S. Eisenstat, H. Walker, Globally convergent inexact Newton methods, SIAM J. Optimization 4 (1994) 393-422.

[33] B. Smith, P. Bjorstad, W. Gropp, Domain Decomposition: Parallel Multilevel methods for elliptic partial differential equations, Cambridge University Press, 1996.

[34] A. Quarteroni, A. Valli, Domain Decomposition Methods for Partial Differential Equations, Oxford University Press, Oxford, 1999.

[35] M. Sala, J. N. Shadid, R. S. Tuminaro, An improved convergence bound for aggregation-based domain decomposition preconditioners, SIAM J. Matrix Analysis 27 (3) (2006) 744-756.

[36] P. T. Lin, M. Sala, J. N. Shadid, R. S. Tuminaro, Performance of fully-coupled algebraic multilevel domain decomposition preconditioners for incompressible flow and transport, submitted to Int. J. Num. Meth. Eng.

[37] N. B. Salah, A. Soulaimani, W. G. Habashi, M. Fortin, A conservative stabilized finite element method for the magento-hydrodyanamics equations, Int. J. Num. Meth. Fuilds 29 (1999) 535-554.

[38] F. Brezzi, On existence, uniqueness and approximation of saddle-point problems arising from Lagrange multipliers, RAIRO Model. Math. Anal. Numer. 21 (1974) 129-151.

[39] M. Gunzburger, Finite Element Methods for Viscous Incompressible Flows, Academic Press, Boston, 1989.

[40] T. Barth, P. Bochev, M. Gunzburger, J. Shadid, A taxonomy of consistently stabilized finite element methods for the Stokes problem, SIAM J. Sci. Comp. 25 (5) (2004) 1585-1607.

[41] F. Brezzi, J. Pitkaranta, On the stabilization of finite element approximations of the stokes problem, Efficient Solutions of Elliptic Systems, Vieweg Notes on Numerical Fluid Mechanics, Vieweg, Wiesbaden, W. Hackbusch (ed.) 10 (1984) 11-19.

[42] N. B. Salah, A. Soulaimani, W. G.Hhabashi, A finite element method for magento-hydrodyanamics, Comp. Meth. Applied Mech. Eng. 190 (2001) 5867-5892.

[43] R. Codina, N. Hernandez-Silva, Stabilized finite element approximation of the stationary magnetohydrodynamics equations, COMPUTATIONAL MECHANICS 38 (4-5) (2006) 344-355.

[44] J.-F. Gerbeau, A stabilized nite element method for the incompressible magnetohydrodynamic equations, Numer. Math. 87 (2000) 83-111.

[45] S. Lankalapalli, J. Flaherty, M. Shephard, H. Strauss, An adaptive nite element method for magnetohydrodynamics, J. Comp. Phys. 225 (2007) 363-381.

[46] T. Hughes, A. N. Brooks, A multidimensional upwind scheme with no cross-wind diffusion, in T.J.R. Hughes (ed.), Finite Element Methods for Convection Dominated Flows, AMD Vol. 34, ASME, New York 19-35. 
[47] A. N. Brooks, T. Hughes, Streamline upwind/petrov-galerkin formulations for convection dominated flows with particular emphasis on the incompressible navier-stokes equations, Comp. Meth. Appl. Mech. and Eng. 32 (1982) 199-259.

[48] T. Hughes, A. Brooks, A theoretical framework for Petrov-Galerkin methods with discontinuous weighting functions: Application to the streamline-upwind procedure, in: R. G. et al (Ed.), Finite Elements in Fluids, Vol. 4, J. Willey \& Sons, 1982, pp. 47-65.

[49] T. J. R. Hughes, L. P. Franca, G. M. Hulbert, A new finite element formulation for computational fluid dynamics: VII. the Galerkin/Least-Squares method for advective-diffusive equations, Computer Methods in Applied Mechanics and Engineering 73 (1989) 173-189.

[50] T. Hughes, L. Franca, M. Balestra, A new finite element formulation for computational fluid dynamics: V. Circumventing the Babuska-Brezzi condition: A stable Petrov-Galerkin formulation of the Stokes problem accommodating equal-order interpolations, Comput. Meth. Appl. Mech. Engrg. 59 (1986) 85-99.

[51] T. Hughes, M. Mallet, A. Mizukami, A new finite element formulation for computational fluid dynamics: II. Beyond SUPG, Comput. Meth. Appl. Mech. Engrg. 54 (1986) 341-355.

[52] F. Shakib, Finite element analysis of the compressible Euler and Navier-Stokes equations, Ph.D. thesis, Division of Applied Mathematics, Stanford University (1989).

[53] S. I. Braginskii, Transport Processes in a Plasma, Reviews of Plasma Physics, Ed. M. A. Leontovich, 1965.

[54] J. D. Jackson, Classical Electrodynamics, 2nd Edition, John Wiley \& Sons, 1975.

[55] A. Dedner, F. Kemm, D. Kroner, C.-D. Munz, T. Schnitzer, M. Wesenberg, Hyperbolic divergence cleaning for the MHD equations, J. Comp. Phys. 175 (2002) 645-673.

[56] L. Chacón, A non-staggered, conservative, $\nabla \cdot \mathbf{B}=\mathbf{0}$, finite-volume scheme for 3D implicit extended magnetohydrodynamics in curvilinear geometries, Comput. Phys. Comm. 163 (2004) 143-171.

[57] R. G. Rehm, H. R. Baum., J. Res. Nat. Bur. Stand 83 (1978) 297-308.

[58] A. J. Majda, J. Sethian, The derivation and numerical solution of the equations for zero mach number combustion, Combust. Sci. Techn. 42 (1985) 185-205.

[59] R. P. Pawlowski, A. G. Salinger, L. A. Romero, J. N. Shadid, Computational design and analysis of movpe reactors, J. Phys. IV France 11 (2001) 197-204.

[60] R. P. Pawlowski, Theodoropoulos, T. J. Mountaziaris, H. K. Moffat, A. G. Salinger, J. N. Shadid, E. J. Thrush, Fundamental models of metalorganic vapor-phase epitaxy of gallium nitride and their use in reactor design, J. Crystal Growth 221 (2000) 622-628.

[61] A. G. Salinger, J. N. Shadid, S. A. Hutchinson, G. L. Hennigan, K. D. Devine, H. K. Moffat, Analysis of gallium arsenide deposition in a horizontal chemical vapor deposition reactor using massively parallel computations, J. Crystal Growth 203 (1999) 516-533.

[62] A. Salinger, R. Pawlowski, J. Shadid, B. van Bloemen Waanders, Computational analysis and optimization of a chemical vapor deposition reactor with large-scale computing, Ind. Eng. Chem. Res. 43 (2004) 4612-4623.

[63] T. Hughes, M. Mallet, A new finite element formulation for computational fluid dynamics: III. The generalized streamline operator for multidimentional advective-diffusive systems, Comput. Meth. Appl. Mech. Engrg. 58 (1986) 305-328. 
[64] T. Hughes, Multiscale phenomena: Green's functions, the dirichlet-to-neumann formulation, subgrid scale models, bubbles and the origins of stabilized methods, Comp. Meth. Appl. Mech. and Eng. 127 (1995) $387-401$.

[65] T. Tezduyar, Stabilibized finite element formulations for incompressible flow calculations, Advances in App. Mech. 28 (1992) 1-44.

[66] T. Tezduyar, Y. J. Park, Discontinuity capturing finite element formulations for nonlinear convectiondiffusion-reaction problems, Comp. Meth. Appl. Mech. Engrg. 59 (1986) 307-325.

[67] R. Codina, Comparison of some finite element methods for solving the diffusion-convection-reaction equations, Comp. Meth. Appl. Mech. and Eng. 156 (1998) 185-210.

[68] E. Burman, A. Ern, Nonlinear diffusion and discrete maximum principle for stabilized galerkin approximations of the convection-diffusion-reaction equation, Comp. Meth. Appl. Mech. and Eng. 191 (2002) 3833-3855.

[69] J. Shadid, A fully-coupled Newton-Krylov solution method for parallel unstructured finite element fluid flow, heat and mass transfer simulations, Int. J. CFD 12 (1999) 199-211.

[70] J. Shadid, R. Tuminaro, K. Devine, G. Henningan, P. Lin, Performance of fully-coupled domain decomposition preconditioners for finite element transport/reaction simulations, J. Comput. Phys. 205 (1) (2005) 24-47.

[71] C. Vincent, R. Boyer, A preconditioned conjugate gradient uzawa-type method for the solution of the Stokes problem bu mixed Q1-P0 stabilized finite elements, Int. J. Numer. Meth. Fluids 14 (1992) 289-298.

[72] S. Wille, A preconditioned alternating inner-outer solution method for the mixed finite element formulation of the Navier-Stokes equations, Int. J. Numer. Meth. Fluids 18 (1994) 1135-1151.

[73] J. E. Dennis, Jr., R. B. Schnabel, Numerical Methods for Unconstrained Optimization and Nonlinear Equations, Series in Automatic Computation, Prentice-Hall, Englewood Cliffs, NJ, 1983.

[74] P. N. Brown, Y. Saad, Hybrid Krylov methods for nonlinear systems of equations, SIAM J. Sci. Stat. Comput. 11 (1990) 450-481.

[75] R. P. Pawlowski, J. N. Shadid, J. P. Simonis, H. F. Walker, Globalaization techniques for Newton-Krylov methods and applications to the fully-coupled solution of the Navier-Stokes equations, SIAM Review 48 (2006) 700-721.

[76] R. S. Dembo, S. C. Eisenstat, T. Steihaug, Inexact Newton methods, SIAM J. Numer. Anal. 19 (1982) 400-408.

[77] S. C. Eisenstat, H. F. Walker, Globally convergent inexact Newton methods, SIAM J. Optimization 4 (1994) 393-422.

[78] S. C. Eisenstat, H. F. Walker, Choosing the forcing terms in an inexact Newton method, SIAM J. Sci. Comput. 17 (1996) 16-32.

[79] J. N. Shadid, R. S. Tuminaro, H. F. Walker, An inexact Newton method for fully-coupled solution of the Navier-Stokes equations with heat and mass transport, J. Comput. Phys. 137 (1997) 155-185.

[80] R. P. Pawlowski, J. N. Shadid, J. P. Simonis, H. F. Walker, Globalization techniques for Newton-Krylov methods and applciations to the fully-coupled solution of the Navier-Stokes equations, submitted to SIAM Review. 
[81] M. Heroux, R. Bartlett, V. Howle, R. Hoekstra, J. Hu, T. Kolda, R. Lehoucq, K. Long, R. Pawlowski, E. Phipps, A. Salinger, H. Thornquist, R. Tuminaro, J. Willenbring, A. Williams, An Overview of Trilinos, Tech. Rep. SAND2003-2927, Sandia National Laboratories (2003).

[82] A. G. Salinger, R. B. Lehoucq, R. P. Pawlowski, J. N. Shadid, Computational bifrucation and stability studies of the 8:1 thermal cavity problem, Int. J. Num. Meth. Fluids 40 (2002) 1059-1073.

[83] R. Lehoucq, A. Salinger, Large-scale eigenvalue calculations for stability analysis of steady flows on massively parallel computers, Int. J. Num. Meth. Fluids 36 (2001) 309-327.

[84] J. N. Shadid, A. G. Salinger, R. P. Pawlowski, P. T. Lin, G. L. Hennigan, R. S. Tuminaro, R. B. Lehoucq, Stabilized FE computational analysis of nonlinear steady state transport/reaction systems, Comp. Meth. Applied Mech. Eng. 195 (2006) 1846-1871.

[85] R. P. Pawlowski, A. G. Salinger, S. J. N., M. T. J., Stability analysis of laminar isothermal impinging jet flows, submitted to Journal of Fluid Mech.

[86] A. G. Salinger, E. A. Burroughs, R. P. Pawlowski, E. T. Phipps, L. A. Romero, Bifurcation tracking algorithms and software for large scale applications, Int. J. Bifurcation ChaosIn press.

[87] Y. Saad, Iterative Methods for Sparse Linear Systems, SIAM, 2003.

[88] K. Devine, E. Boman, R. Heaphy, B. Hendrickson, C. Vaughan, Zoltan data management services for parallel dynamic applications, Computing in Science and Engineering 4 (2) (2002) 90-97.

[89] O. Axelsson, Iterative Solution Methods, Cambridge University Press, New York, 1994.

[90] M. Sala, M. Heroux, Robust algebraic preconditioners with IFPACK 3.0, Tech. Rep. SAND2005-0662, Sandia National Laboratories, Albuquerque, NM, 87185 (2005).

[91] R. S. Tuminaro, M. Heroux, S. A. Hutchinson, J. N. Shadid, Aztec user's guide-version 2.1, Tech. Rep. Sand99-8801J, Sandia National Laboratories, Albuquerque NM, 87185 (Nov. 1999).

[92] R. Tuminaro, C. Tong, J. Shadid, K.D.Devine, D. Day, On a multilevel preconditioning module for unstructured mesh Krylov solvers: two-level Schwarz, Comm. Num. Method. Eng. 18 (2002) 383-389.

[93] U. Trottenberg, C. Oosterlee, A. Schüller, Multigrid, Academic Press, London, 2001.

[94] W. L. Briggs, V. E. Henson, S. McCormick, A Multigrid Tutorial, Second Edition, SIAM, Philadelphia, 2000 .

[95] J. Ruge, K. Stüben, Algebraic multigrid (AMG), in: S. F. McCormick (Ed.), Multigrid Methods, Vol. 3 of Frontiers in Applied Mathematics, SIAM, Philadelphia, PA, 1987, pp. 73-130.

[96] A. Brandt, S. McCormick, J. Ruge, Algebraic multigrid (AMG) for sparse matrix equations, in: D. J. Evans (Ed.), Sparsity and Its Applications, Cambridge University Press, Cambridge, 1984.

[97] P. Vaněk, J. Mandel, M. Brezina, Algebraic multigrid by smoothed aggregation for second and fourth order elliptic problems, Computing 56 (1996) 179-196.

[98] P. Vaněk, M. Brezina, J. Mandel, Convergence of algebraic multigrid based on smoothed aggregation, Numerische Mathematik 88 (2001) 559-579.

[99] G. Karypis, V. Kumar, Parallel multilevel k-way partitioning scheme for irregular graphs, ACM/IEEE Proceedings of SC96: High Performance Networking and Computing.

[100] M. Sala, J. Hu, R. Tuminaro, ML 3.1 smoothed aggregation user's guide, Tech. Rep. SAND2004-4819, Sandia National Laboratories (September 2004). 
[101] T. Davis, Direct methods for sparse linear systems, SIAM, Philadelphia, PA, 2006.

[102] M. Sala, Amesos 2.0 reference guide, Tech. Rep. SAND2004-4820, Sandia National Laboratories (September 2004).

[103] M. Sala, R. Tuminaro, A new Petrov-Galerkin smoothed aggregation preconditioner for nonsymmetric linear systems, SIAM J. Sci. Comput. 31 (1) (2008) 143-166.

[104] C. Lasser, A. Toselli, Convergence of some two-level overlapping domain decomposition preconditioners with smoothed aggregation coarse spaces, in: L. Pavarino, A. Toselli (Eds.), Recent Developments in Domain Decomposition Methods, Vol. 23 of LNCSE, Springer Verlag, 2002.

[105] M. Gee, C. Siefert, J. Hu, R. Tuminaro, M. Sala, ML 5.0 smoothed aggregation user's guide, Tech. Rep. SAND2006-2649, Sandia National Laboratories, Albuquerque, NM, 87185 (2006).

[106] P. T. Lin, M. Sala, J. N. Shadid, R. S. Tuminaro, Performance of a geometric and an algebraic multilevel preconditioner for incompressible flow and transport, in: Computational Mechanics: WCCM VI in conjunction with APCOM'04, Tsinghus Univ. Press, Springer-Verlag, 2004.

[107] P. T. Lin, M. Sala, J. N. Shadid, R. S. Tuminaro, Performance of fully-coupled algebraic multilevel domain decomposition preconditioners for incompressible flow and transport, Int. J. Num. Meth. Eng. 67 (9) (2006) 208-225.

[108] P. T. Lin, J. N. Shadid, M. Sala, R. Tuminaro, G. L. Hennigan, R. J. Hoekstra, Performance of a parallel algebraic multilevel preconditioner for stabilized finite element semiconductor device modeling, J. Comp. Phys. (2009) In revision.

[109] H. K. Moffat, Magnetic Field Generation in Electrically Conducting Fluids, Cambridge Univ. Press, 1983.

[110] H. K. Moffat, magneto-hydrodynamics, Kluwer, Dordrecht, 1990.

[111] R. Codina, N. Hernadez-Silva, Stabilized finite element approximation of the stationary magentohydrodyanamics equations, Comput. Mech. 38 (2006) 344-355.

[112] G. K. Batchelor, An Introduction to Fluid Mechanics, Cambridge Univ. Press, 1967.

[113] V. M. Fadeev, I. F. Kvartskhava, N. N. Komarov, Self-focusing of local plasma currents., Nuclear fusion 5 (3) (1965) $202-209$.

[114] M. Hughes, K. A. Pericleous, M. Cross, The numerical modelling of dc electromagnetic pump and brake flow, Appl. Math. Modelling 19 (1995) 713-723.

[115] S. L. L. Verardi, J. R. Cardoso, M. C. Costa, Three-dimensional finite element analysis of mhd duct flow by the penalty function formulation, IEEE TRANSACTIONS ON MAGNETICS 37 (5) (2001) 3384-3387.

[116] N. B. Morley, M. S. Tillack, Magnetohydrodynamics, chapter in the Standard Handbook for Electrical Engineers, 15th Edition, McGraw Hill, 2006.

[117] X.-C. Cai, An additive schwarz algorithm for nonselfadjoint elliptic equations, in: T. Chan, R. G., J. P., O. B. Widlund (Eds.), Third International Symposium on Domain Decomposition Methods for Partial Differential Equations, 1989, pp. 232-244.

[118] X.-C. Cai, W. D. Gropp, D. E. Keyes, Convergence rate estimate for a domain decomposition method, Numer. Math. 61 (2) (1992) 153-169.

[119] C. Farhat, N. Maman, G. Brown, Mesh partitioning for implicit computations via iterative domain decomposition - impact and optimization of the subdomain aspect ratio, Int. J. Num. Meth. Eng. 38 (6) (1995) 989-1000. 
[120] B. A. Hendrickson, Load balancing fictions, falsehoods and fallacies, Applied Mathematical Modelling 25 (2) (2000) 99-108.

[121] B. A. Hendrickson, T. G. Kolda, Graph partitioning models for parallel computing, Parallel Computing 26 (12) (2000) 1519-1534.

[122] M. Benzi, Preconditioning techniques for large linear systems: A survey, J. Comp. Physics 182 (2002) 418-477.

[123] W. Gropp, D. Kaushik, D. Keyes, B. Smith, High-performance parallel implicit cfd, Parallel Computing 27 (2001) 337-362.

[124] R. Tuminaro, C. Tong, J. Shadid, K.D.Devine, D. Day, On a multilevel preconditioning module for unstructured mesh Krylov solvers: two-level schwarz, Comm. Num. Method. Eng. 18 (2002) 383-389.

[125] S. Chandrasekhar, Hydrodynamic and Hydromagnetic Stability, Oxford Univ. Press, 1961.

[126] D. Biskamp, Magnetic Reconnection in Plasmas, Cambridge University Press, Cambridge, UK, 2000.

[127] D. A. Knoll, L. Chacón, Coalescence of magnetic islands, sloshing, and the pressure problem., Phys. Plasmas 13 (3) (2006) $32307-1$.

[128] E. Priest, T. Forbes, Magnetic Reconnection: MHD Theory and Applications, Cambridge Univ. Press, 2006. 